\title{
A Beurling-Blecher-Labuschagne theorem for noncommutative Hardy spaces associated with semifinite von Neumann algebras
}

\author{
Lauren Sager
}

\begin{abstract}
In 2008, Blecher and Labuschagne extended Beurling's classical theorem to $H^{\infty}$ invariant subspaces of $L^{p}(\mathcal{M}, \tau)$ for a finite von Neumann algebra $\mathcal{M}$ with a finite, faithful, normal tracial state $\tau$ when $1 \leq p \leq \infty$. In this paper, using Arveson's non-commutative Hardy space $H^{\infty}$ in relation to a von Neumann algebra $\mathcal{M}$ with a semifinite, faithful, normal tracial weight $\tau$, we prove a Beurling-Blecher-Labuschagne theorem for $H^{\infty}$-invariant spaces of $L^{p}(\mathcal{M}, \tau)$ when $0<p \leq \infty$. The proof of the main result relies on proofs of density theorems for $L^{p}(\mathcal{M}, \tau)$ and semifinite versions of several other known theorems from the finite case. Using the main result, we are able to completely characterize all $H^{\infty}$-invariant subspaces of $L^{p}\left(\mathcal{M} \rtimes_{\alpha} \mathbb{Z}, \tau\right)$, where $\mathcal{M} \rtimes_{\alpha} \mathbb{Z}$ is a crossed product of a semifinite von Neumann algebra $\mathcal{M}$ by the integer group $\mathbb{Z}$ and $H^{\infty}$ is a non-selfadjoint crossed product of $\mathcal{M}$ by $\mathbb{Z}^{+}$. As an example, we characterize all $H^{\infty}$-invariant subspaces of the Schatten $p$-class $S^{p}(\mathcal{H})$, where $H^{\infty}$ is the lower triangular subalgebra of $B(\mathcal{H})$, for each $0<p \leq \infty$.
\end{abstract}

\section{Introduction}

Let $\mathcal{H}$ be an infinite dimensional separable Hilbert space with an orthonormal base $\left\{e_{m}\right\}_{m \in \mathbb{Z}}$, and $B(\mathcal{H})$ be the set of all bounded linear operators on $\mathcal{H}$. Let $\tau=\operatorname{Tr}$ be the usual trace on $B(\mathcal{H})$, i.e.

$$
\tau(x)=\sum_{i \in \mathbb{Z}}\left\langle x e_{m}, e_{m}\right\rangle, \quad \text { for all positive } x \text { in } B(\mathcal{H}) .
$$

For each $0<p<\infty$, the Schatten $p$-class $S^{p}(\mathcal{H})$ consists all these elements $x$ in $B(\mathcal{H})$ such that $\tau\left(|x|^{p}\right)<\infty$. It is well-known that $S^{p}(\mathcal{H})$ is a complete metric space (a Banach space when $p \geq 1$ and a Hilbert space when $p=2$ ). Moreover, $S^{p}(\mathcal{H})$ is a two sided ideal of $B(\mathcal{H})$.

Let

$$
\mathcal{A}=\left\{x \in B(\mathcal{H}):\left\langle x e_{m}, e_{n}\right\rangle=0, \quad \forall n<m\right\}
$$

2000 Mathematics Subject Classification. Primary 32A35, 46L52; Secondary 47A15, 47L65.

Key words and phrases. Beurling's theorem, Schatten p-classes, semifinite von Neuman algebra, noncommutative Hardy space, crossed products of von Neumann algebras, $L^{p}$ spaces, invariant subspaces. 
be the lower triangular subalgebra of $B(\mathcal{H})$. In the paper, we are interested in answering the following question, which is implicitly asked by McAsey, Muhly and Saito in Example 2.6 of 25 .

Problem 1.1. Given a closed subspace $\mathcal{K}$ of the Schatten $p$-class $S^{p}(\mathcal{H})$ where $0<p<\infty$, such that $\mathcal{K}$ satisfies $\mathcal{A} \mathcal{K} \subseteq \mathcal{K}$, how can we characterize the subspace $\mathcal{K}$ ?

The answer to Problem 1.1 is closely related to our generalization of the classical Beurling theorem for a Hardy space. Recall the classical Beurling theorem for invariant subspaces as follows. Let $\mathbb{T}$ be the unit circle, and let $\mu$ be the measure on $\mathbb{T}$ such that $d \mu=\frac{1}{2 \pi} d \theta$. Let $L^{\infty}(\mathbb{T}, \mu)$ be the commutative von Neumann algebra on $\mathbb{T}$, and define $L^{2}(\mathbb{T}, \mu)$ to be the closure of $L^{\infty}(\mathbb{T}, \mu)$ under the $\|\cdot\|_{2}$. Then $L^{2}(\mathbb{T}, \tau)$ is a Hilbert space with orthonormal basis $\left\{z^{n}: n \in \mathbb{Z}\right\}$. Let $H^{2}=\overline{\operatorname{span}\left\{z^{n}: n \geq 0\right\}}{ }^{\|\cdot\|_{2}} \subseteq L^{2}\left((\mathbb{T}, \mu)\right.$ and $H^{\infty}=H^{2} \cap L^{\infty}(\mathbb{T}, \mu)$. If we define $M_{\psi}(f)=\psi f$ for every $f \in L^{2}(\mathbb{T}, \mu)$, it is easy to show that $L^{\infty}(\mathbb{T}, \mu)$ has a representation onto $\mathcal{B}\left(L^{2}(\mathbb{T}, \mu)\right)$ by the map $\psi \rightarrow M_{\psi}$. Therefore, $L^{\infty}(\mathbb{T}, \mu)$ and $H^{\infty}$ can be assumed to act naturally on $L^{2}(\mathbb{T}, \mu)$ by multiplication on the left (or right). The classical Beurling's theorem, proven in 1949 by A. Beurling in [6], states the following: If $\mathcal{W}$ is a nonzero closed, $H^{\infty}$-invariant subspace (or, equivalently, $z \mathcal{W} \subseteq \mathcal{W}$ for every $\left.z \in H^{\infty}\right)$ of $H^{2}$, then $\mathcal{W}=\psi H^{2}$ for some $\psi \in H^{\infty}$ with $|\psi|=1$ a.e. $(\mu)$.

Then we define $L^{p}(\mathbb{T}, \tau)$ to be the closure of $L^{\infty}(\mathbb{T}, \tau)$ under $\|\cdot\|_{p}$, and $H^{p}=\left\{f \in L^{p}(\mathbb{T}, \mu)\right.$ : $\int_{\mathbb{T}} f\left(e^{i \theta}\right) e^{i n \theta} d \mu(\theta)=0$ for $\left.n \in \mathbb{N}\right\}$ for $1 \leq p<\infty$. Beurling's theorem has been extended for $H^{\infty}$-invariant subspaces in Hardy spaces $H^{p}$ for $1 \leq p \leq \infty$. (See, for example, [8], [15], [16], [17], [18], [35], and others). The classical Beurling's theorem has been extended in many other ways as well.

One such extension comes from the work of D. Blecher and L. Labuschagne in [7]. We recall the construction of $L^{p}(\mathcal{M}, \tau)$. Let $\mathcal{M}$ be a semifinite von Neumann algebra, and let $\tau$ be a faithful, normal tracial weight on $\mathcal{M}$ (when $\tau(I)<\infty, \mathcal{M}$ is finite). Let $\mathcal{I}$ be the set of elementary operators in $\mathcal{M}$ (when $\mathcal{M}$ is finite, $\mathcal{I}=\mathcal{M}$ ). Then define a mapping from $\mathcal{I}$ to $[0, \infty)$ by $\|x\|_{p}=\left(\tau\left(|x|^{p}\right)\right)^{1 / p}$ for every $x \in \mathcal{I}$, and where $|x|=\sqrt{x^{*} x}$. It is nontrivial to prove that when $1 \leq p<\infty,\|\cdot\|_{p}$ defines a norm on $\mathcal{I}$, which we call the p-norm. We may then define $L^{p}(\mathcal{M}, \tau)=\overline{\mathcal{I}}^{\|\cdot\|_{p}}$. We let $L^{\infty}(\mathcal{M}, \tau)=\mathcal{M}$, and this space acts naturally on $L^{p}(\mathcal{M}, \tau)$ by left (or right) multiplication.

We then recall the definition of Arveson's non-commutative Hardy space from [1]. If $\mathcal{M}$ is a von Neumann algebra, with faithful, normal tracial weight $\tau$, let $\mathcal{A} \subseteq \mathcal{M}$ be a weak* closed subalgebra. Then let $\mathcal{D}=\mathcal{A} \cap \mathcal{A}^{*}$ be a von Neumann subalgebra of $\mathcal{M}$. Then there exists $\Phi: \mathcal{M} \rightarrow \mathcal{D}$, a faithful, normal, trace-preserving conditional expectation, which can be extended to $\Phi: L^{1}(\mathcal{M}, \tau) \rightarrow L^{1}(\mathcal{D}, \tau)$. Then $\mathcal{A}$ is called a non-commutative Hardy space if (1) $\Phi(x y)=\Phi(x) \Phi(y)$ for every $x, y \in \mathcal{A} ;(2) \mathcal{A}+\mathcal{A}^{*}$ is weak ${ }^{*}$ dense in $\mathcal{M} ;(3) \tau(\Phi(x))=\tau(x)$ for every $x \in \mathcal{M}$.

Blecher and Labuschagne proved the following theorem for finite von Neumann algebras in [7. Let $\mathcal{M}$ be a finite von Neumann algebra with a faithful, tracial, normal state $\tau$, and $H^{\infty}$ be a maximal subdiagonal subalgebra of $\mathcal{M}$ with $\mathcal{D}=H^{\infty} \cap\left(H^{\infty}\right)^{*}$. Suppose that $\mathcal{K}$ is a closed 
$H^{\infty}$-right-invariant subspace of $L^{p}(\mathcal{M}, \tau)$, for some $1 \leq p \leq \infty$. (For $p=\infty$ it is assumed that $\mathcal{K}$ is weak* closed.) Then $\mathcal{K}$ may be written as a column $L^{p}$-sum $\mathcal{K}=\mathcal{Z} \oplus^{\text {col }}\left(\oplus_{i}^{\text {col }} u_{i} H^{p}\right)$, where $\mathcal{Z}$ is a closed (indeed weak ${ }^{*}$ closed if $p=\infty$ ) subspace of $L^{p}(\mathcal{M}, \tau)$ such that $\mathcal{Z}=\left[\mathcal{Z} H_{0}^{\infty}\right]_{p}$, and where $u_{i}$ are partial isometries in $\mathcal{M} \cap \mathcal{K}$ satisfying certain conditions (For more details, see [7.) Here $\oplus_{i}^{\text {col }} u_{i} H^{p}$ and $\mathcal{Z}=\left[\mathcal{Z} H_{0}^{\infty}\right]_{p}$ are of type 1 , and type 2 respectively (also see [26] for definitions of invariant subspaces of different types).

Examples of finite von Neumann algebras include the spaces $M_{n}(\mathbb{C})$ of all $n \times n$ matrices with complex entries when $1 \leq n<\infty$. However, if $\mathcal{H}$ is an infinite dimensional separable Hilbert space and we view $B(\mathcal{H})$ as $M_{\infty}(\mathbb{C})$, the set of all (bounded) $\infty \times \infty$ matrices with complex entries, then $B(\mathcal{H})$ is a semifinite von Neumann algebra, and no longer satisfies the hypothesis of the Beurling-Blecher-Labuschagne theorem.

In this paper, we therefore consider a version of Blecher and Labuschagne's Beurling's theorem for semifinite von Neumann algebras. We seek to characterize $H^{\infty}$-invariant spaces of $L^{p}(\mathcal{M}, \tau)$ spaces. Adapting Blecher and Labuschagne's theorem to the semifinite case, we prove the following result:

Theorem 4.6. Let $1 \leq p<\infty$. Let $\mathcal{M}$ be a von Neumann algebra with a faithful, normal, semifinite tracial weight $\tau$, and $H^{\infty}$ be a semifinite subdigonal subalgebra of $\mathcal{M}$ (see Definition 2.7). Let $\mathcal{D}=H^{\infty} \cap\left(H^{\infty}\right)^{*}$. Assume that $\mathcal{K}$ is a closed subspace of $L^{p}(\mathcal{M}, \tau)$ such that $H^{\infty} \mathcal{K} \subseteq \mathcal{K}$.

Then there exist a closed subspace $Y$ of $L^{p}(\mathcal{M}, \tau)$ and a family $\left\{u_{\lambda}\right\}_{\lambda \in \Lambda}$ of partial isometries in $\mathcal{M}$ such that:

(i) $u_{\lambda} Y^{*}=0$ for all $\lambda \in \Lambda$.

(ii) $u_{\lambda} u_{\lambda}^{*} \in \mathcal{D}$ and $u_{\lambda} u_{\mu}^{*}=0$ for all $\lambda, \mu \in \Lambda$ with $\lambda \neq \mu$.

(iii) $Y=\left[H_{0}^{\infty} Y\right]_{p}$.

(iv) $\mathcal{K}=Y \oplus^{\text {row }}\left(\oplus_{\lambda \in \Lambda}^{\text {row }} H^{p} u_{\lambda}\right)$

Here $\oplus^{\text {row }}$ is the row sum of subspaces defined in Definition 2.15.

However, many of the methods used by Blecher and Labuschagne do not apply directly when $\mathcal{M}$ is a semifinite von Neumann algebra. Thus, we prove a density theorem for semifinite von Neumann algebras through a series of lemmas and propositions.

Proposition 4.1. Let $\mathcal{M}$ be a von Neumann algebra with a faithful, normal, semifinite tracial weight $\tau$, and $H^{\infty}$ be a semifinite subdigonal subalgebra of $\mathcal{M}$. Let $1 \leq p<\infty$. Assume that $\mathcal{K}$ is a closed subspace in $L^{p}(\mathcal{M}, \tau)$ such that $H^{\infty} \mathcal{K} \subseteq \mathcal{K}$. Then the following statements are true.

(i) $\mathcal{K} \cap \mathcal{M}={\overline{\mathcal{K} \cap \mathcal{M}^{w}}}^{w^{*}} \cap L^{p}(\mathcal{M}, \tau)$.

(ii) $\mathcal{K}=[\mathcal{K} \cap \mathcal{M}]_{p}$.

Proposition 4.2. Let $\mathcal{M}$ be a von Neumann algebra with a faithful, normal, semifinite tracial weight $\tau$, and $H^{\infty}$ be a semifinite subdigonal subalgebra of $\mathcal{M}$.

Assume that $\mathcal{K} \subseteq \mathcal{M}$ is weak ${ }^{*}$-closed subspace such that $H^{\infty} \mathcal{K} \subseteq \mathcal{K}$. Then

$$
\mathcal{K}=\overline{\left[\mathcal{K} \cap L^{p}(\mathcal{M}, \tau)\right]_{p} \cap \mathcal{M}^{w^{*}}}, \quad \forall 1 \leq p<\infty .
$$


Proposition 4.4. Let $\mathcal{M}$ be a von Neumann algebra with a faithful, normal, semifinite tracial weight $\tau$, and $H^{\infty}$ be a semifinite subdigonal subalgebra of $\mathcal{M}$. Assume that $S \subseteq \mathcal{M}$ is a subspace such that $H^{\infty} S \subseteq S$. Then

$$
\left[S \cap L^{p}(\mathcal{M}, \tau)\right]_{p}=\left[\bar{S}^{w^{*}} \cap L^{p}(\mathcal{M}, \tau)\right]_{p}, \quad \forall 1 \leq p<\infty .
$$

Subsequently, we are able to prove a noncommutative Beurling-Blecher-Labuschagne theorem for the semifinite case when $0<p<1$.

Theorem 5.4. Let $0<p<1$. Let $\mathcal{M}$ be a von Neumann algebra with a faithful, normal, semifinite tracial weight $\tau$, and $H^{\infty}$ be a semifinite subdigonal subalgebra of $\mathcal{M}$ (see Definition 2.7). Let $\mathcal{D}=H^{\infty} \cap\left(H^{\infty}\right)^{*}$. Assume that $\mathcal{K}$ is a closed subspace of $L^{p}(\mathcal{M}, \tau)$ such that $H^{\infty} \mathcal{K} \subseteq \mathcal{K}$.

Then there exist a closed subspace $Y$ of $L^{p}(\mathcal{M}, \tau)$ and a family $\left\{u_{\lambda}\right\}_{\lambda \in \Lambda}$ of partial isometries in $\mathcal{M}$ such that:

(i) $u_{\lambda} Y^{*}=0$ for all $\lambda \in \Lambda$.

(ii) $u_{\lambda} u_{\lambda}^{*} \in \mathcal{D}$ and $u_{\lambda} u_{\mu}^{*}=0$ for all $\lambda, \mu \in \Lambda$ with $\lambda \neq \mu$.

(iii) $Y=\left[H_{0}^{\infty} Y\right]_{p}$.

(iv) $\mathcal{K}=Y \oplus^{\text {row }}\left(\oplus_{\lambda \in \Lambda}^{\text {row }} H^{p} u_{\lambda}\right)$

Here $\oplus^{\text {row }}$ is the row sum of subspaces defined in Definition 2.15.

Here, we use similar methods to our proof for $1 \leq p<\infty$, including proving a similar density theorem (see Proposition 5.1, Proposition 5.2).

Using theorems 4.6 and 5.4 and corollary 5.5, we are then able to prove a Beurling-BlecherLabuschagne-like theorem for the crossed product of a semifinite von Neumann algebra $\mathcal{M}$ by a trace-preserving action $\alpha$ when $0<p<\infty$. We are actually able to fully characterize the $H^{\infty}$-invariant subspace of the crossed product.

THEOREM 6.3. Let $\mathcal{M}$ be a von Neumann algebra with a semifinite, faithful, normal, tracial weight $\tau$, and $\alpha$ be a trace-preserving *-automorphism of $\mathcal{M}$. Denote by $\mathcal{M} \rtimes_{\alpha} \mathbb{Z}$ the crossed product of $\mathcal{M}$ by an action $\alpha$, and still denote by $\tau$ a semifinite, faithful, normal, extended tracial weight on $\mathcal{M} \rtimes_{\alpha} \mathbb{Z}$.

Let $H^{\infty}$, a weak $*$-closed nonself-adjoint subalgebra generated by $\{\Lambda(n) \Psi(x): x \in \mathcal{M}, n \geq 0\}$ in $\mathcal{M} \rtimes_{\alpha} \mathbb{Z}$, be a semifinite subdiagonal subalgebra of $\mathcal{M} \rtimes_{\alpha} \mathbb{Z}$. Then the following statements are true.

(i) Let $0<p<\infty$. Assume that $\mathcal{K}$ is a closed subspace of $L^{p}\left(\mathcal{M} \rtimes_{\alpha} \mathbb{Z}, \tau\right)$ such that $H^{\infty} \mathcal{K} \subseteq \mathcal{K}$. Then there exist a projection $q$ in $\mathcal{M}$ and a family $\left\{u_{\lambda}\right\}_{\lambda \in \Lambda}$ of partial isometries in $\mathcal{M} \rtimes_{\alpha} \mathbb{Z}$ satisfying

(a) $u_{\lambda} q=0$ for all $\lambda \in \Lambda$;

(b) $u_{\lambda} u_{\lambda}^{*} \in \mathcal{M}$ and $u_{\lambda} u_{\mu}^{*}=0$ for all $\lambda, \mu \in \Lambda$ with $\lambda \neq \mu$;

(c) $\mathcal{K}=\left(L^{p}\left(\mathcal{M} \rtimes_{\alpha} \mathbb{Z}, \tau\right) q\right) \oplus^{\text {row }}\left(\oplus_{\lambda \in \Lambda}^{\text {row }} H^{p} u_{\lambda}\right)$.

(ii) Assume that $\mathcal{K}$ is a weak *-closed subspace of $\mathcal{M} \rtimes_{\alpha} \mathbb{Z}$ such that $H^{\infty} \mathcal{K} \subseteq \mathcal{K}$. Then there exist a projection $q$ in $\mathcal{M}$ and a family $\left\{u_{\lambda}\right\}_{\lambda \in \Lambda}$ of partial isometries in $\mathcal{M} \rtimes_{\alpha} \mathbb{Z}$ satisfying 
(a) $u_{\lambda} q=0$ for all $\lambda \in \Lambda$;

(b) $u_{\lambda} u_{\lambda}^{*} \in \mathcal{M}$ and $u_{\lambda} u_{\mu}^{*}=0$ for all $\lambda, \mu \in \Lambda$ with $\lambda \neq \mu$;

(c) $\mathcal{K}=\left(\left(\mathcal{M} \rtimes_{\alpha} \mathbb{Z}\right) q\right) \oplus^{\text {row }}\left(\oplus_{\lambda \in \Lambda}^{\text {row }} H^{\infty} u_{\lambda}\right)$.

In 25, McAsey, Muhly and Saito prove a Beurling's theorem for a crossed product. Suppose $\mathcal{M}$ is a finite von Neumann algebra with finite trace $\tau$ and $\alpha$, a trace preserving automorphism of $\mathcal{M}$, such that $\alpha$ fixes each element of the center $Z(\mathcal{M})$ of $\mathcal{M}$. Then let $\mathcal{A}=\mathcal{M} \rtimes_{\alpha} \mathbb{Z}_{+}$. Then every $\mathcal{A}$ and $\mathcal{A}^{*}$-invariant subspace $\mathcal{K}$ of $L^{2}(M, \tau)$ has the form $\mathcal{K}=v H^{2}$ for a partial isometry $v$ in the commutant of right multiplication by $\mathcal{M}$ on $L^{2}(\mathcal{M}, \tau)$. This follows from theorem 6.3 when $\tau$ is finite, and $p=2$.

McAsey, Muhly and Saito's result is a corollary of a result by Nazaki and Watatani in [26]. Suppose $\mathcal{M}$ is a finite von Neumann algebra with trace $\tau, \mathcal{D} \subseteq \mathcal{M}$ and a faithful, normal, trace-preserving conditional expectation $\Phi: \mathcal{M} \rightarrow \mathcal{D}$. We let $H^{\infty}$ be a maximal subdiagonal algebra with respect to $\Phi$, and suppose that $Z(\mathcal{D}) \subseteq Z(\mathcal{M})$. Then, if we let $\mathcal{K}$ be a $H^{\infty}$-invariant subspace of $L^{2}(\mathcal{M}, \tau)$ such that $\mathcal{K}$ is of $H^{\infty}$-type $I$ (in the sense defined in [26]), there exists a partial isometry $v$ in the commutant of right multiplcation by $\mathcal{M}$ such that $\mathcal{K}=v H^{2}$. Again, this follows from our result in the finite case when $p=2$.

Similarly, Saito in 32 proves another Beurling-like theorem for a finite von Neumann algebra $\mathcal{M}$. Let a closed $\mathcal{K}$ of $L^{2}(\mathcal{M}, \tau)$ invariant under $\mathcal{M} \rtimes_{\alpha} \mathbb{Z}_{+}$such that there are no subspaces of $\mathcal{K}$ with $\mathcal{M} \rtimes_{\alpha} \mathbb{Z} \mathcal{K} \subseteq \mathcal{K}$ such that $\mathcal{K}$ has the form $\sum_{n=0}^{\infty} \oplus V_{n} H^{2}$ with $\left\{V_{n}\right\}$ a family of partial isometries with $\left\{V_{n} v_{n}^{*}\right\}$ is mutually orthonogal.

We are also able to prove a Beurling-Blecher-Labuschagne-like theorem for the Schatten $p$ classes for $0<p<\infty$, as described at the beginning of this section, using theorems 4.6, 5.4 and corollary 5.5 .

Corollary 6.4. Let $\mathcal{H}$ be a separable Hilbert space with an orthonormal base $\left\{e_{m}\right\}_{m \in \mathbb{Z}}$. Let $H^{\infty}$ be the lower triangular subalgebra of $B(\mathcal{H})$, i.e.

$$
H^{\infty}=\left\{x \in B(\mathcal{H}):\left\langle x e_{m}, e_{n}\right\rangle=0, \quad \forall n<m\right\} .
$$

Let $\mathcal{D}=H^{\infty} \cap\left(H^{\infty}\right)^{*}$ be the diagonal subalgebra of $B(\mathcal{H})$.

(i) For each $0<p<\infty$, let $S^{p}(\mathcal{H})$ be the Schatten $p$-class. Assume that $\mathcal{K}$ is a closed subspace of $S^{p}(\mathcal{H})$ such that $H^{\infty} \mathcal{K} \subseteq \mathcal{K}$. Then there exist a projection $q$ in $\mathcal{D}$ and a family $\left\{u_{\lambda}\right\}_{\lambda \in \Lambda}$ of partial isometries in $B(\mathcal{H})$ satisfying

(a) $u_{\lambda} q=0$ for all $\lambda \in \Lambda$;

(b) $u_{\lambda} u_{\lambda}^{*} \in \mathcal{D}$ and $u_{\lambda} u_{\mu}^{*}=0$ for all $\lambda, \mu \in \Lambda$ with $\lambda \neq \mu$;

(c) $\mathcal{K}=\left(S^{p}(\mathcal{H}) q\right) \oplus^{\text {row }}\left(\oplus_{\lambda \in \Lambda}^{\text {row }} H^{p} u_{\lambda}\right)$.

(ii) Assume that $\mathcal{K}$ is a weak $*$-closed subspace of $B(\mathcal{H})$ such that $H^{\infty} \mathcal{K} \subseteq \mathcal{K}$. Then there exist a projection $q$ in $\mathcal{D}$ and a family $\left\{u_{\lambda}\right\}_{\lambda \in \Lambda}$ of partial isometries in $B(\mathcal{H})$ satisfying

(a) $u_{\lambda} q=0$ for all $\lambda \in \Lambda$;

(b) $u_{\lambda} u_{\lambda}^{*} \in \mathcal{D}$ and $u_{\lambda} u_{\mu}^{*}=0$ for all $\lambda, \mu \in \Lambda$ with $\lambda \neq \mu$;

(c) $\mathcal{K}=(B(\mathcal{H}) q) \oplus^{\text {row }}\left(\oplus_{\lambda \in \Lambda}^{\text {row }} H^{\infty} u_{\lambda}\right)$. 
However, if we have that this projection $q$ in $\mathcal{D}$ has the characteristic that $S^{p}(\mathcal{H}) q \subseteq H^{p}$, then we can prove corollary 6.6, and therefore fully characterize a $H^{\infty}$-invariant subspace $\mathcal{K} \subseteq H^{p}$ when $0<p \leq \infty$ and $\mathcal{H}$ is a separable Hilbert space with an orthonormal base.

Corollary 6.6. Let $\mathcal{H}$ be a separable Hilbert space with an orthonormal base $\left\{e_{m}\right\}_{m \in \mathbb{Z}}$. Let $H^{\infty}$ be the lower triangular subalgebra of $B(\mathcal{H})$, i.e.

$$
H^{\infty}=\left\{x \in B(\mathcal{H}):\left\langle x e_{m}, e_{n}\right\rangle=0, \quad \forall n<m\right\} .
$$

Let $\mathcal{D}=H^{\infty} \cap\left(H^{\infty}\right)^{*}$ be the diagonal subalgebra of $B(\mathcal{H})$.

(i) For each $0<p<\infty$, if $\mathcal{K}$ is a closed subspace of $H^{p}$ such that $H^{\infty} \mathcal{K} \subseteq \mathcal{K}$, then there exists a family $\left\{u_{\lambda}\right\}_{\lambda \in \Lambda}$ of partial isometries in $H^{\infty}$ satisfying

(a) $u_{\lambda} u_{\lambda}^{*} \in \mathcal{D}$ and $u_{\lambda} u_{\mu}^{*}=0$ for all $\lambda, \mu \in \Lambda$ with $\lambda \neq \mu$;

(b) $\mathcal{K}=\oplus_{\lambda \in \Lambda}^{\text {row }} H^{p} u_{\lambda}$.

(ii) Assume that $\mathcal{K}$ is a weak *-closed subspace of $H^{\infty}$ such that $H^{\infty} \mathcal{K} \subseteq \mathcal{K}$. Then there exists a family $\left\{u_{\lambda}\right\}_{\lambda \in \Lambda}$ of partial isometries in $H^{\infty}$ satisfying

(a) $u_{\lambda} u_{\lambda}^{*} \in \mathcal{D}$ and $u_{\lambda} u_{\mu}^{*}=0$ for all $\lambda, \mu \in \Lambda$ with $\lambda \neq \mu$;

(b) $\mathcal{K}=\oplus_{\lambda \in \Lambda}^{\text {row }} H^{\infty} u_{\lambda}$.

Therefore, we are able to answer the question given in problem 1.1 and fully characterize an $\mathcal{A}$-invariant subspace of a Schatten $p$-class: given a subpace $\mathcal{K} \subseteq S^{p}(\mathcal{H})$ such that $\mathcal{A K} \subseteq \mathcal{K}$, we have that $\mathcal{K}=\left(S^{p}(\mathcal{H}) q\right) \oplus_{\lambda \in \Lambda}^{\text {row }} H^{p} u_{\lambda}$ when $0<p<\infty$, and $\mathcal{K}=(B(\mathcal{H}) q) \oplus^{\text {row }}\left(\oplus_{\lambda \in \Lambda}^{\text {row }} H^{\infty} u_{\lambda}\right)$ when $p=\infty$.

The outline of the paper is as follows. In section 2, we discuss preliminary definitions and notations. In section 3, we prove the Beurling-Blecher-Labuschagne theorem for $L^{p}(\mathcal{M}, \tau)$ when $p=2$, and extend this theorem in section 4 to the case when $1 \leq p \leq \infty$. We provide several more preliminaries and further extend the Beurling-Blecher-Labuschagne theorem to $L^{p}(M, \tau)$ for $0<p<1$ in section 5. Finally, in section 6, we discuss some applications of our results on invariant subspaces for analytic crossed products and discuss the results for the Schatten $p$-class.

\section{Preliminaries and Notation}

In this section we give some preliminary definitions and results for non-commutative $L^{p}$ spaces for a von Neumann algebra with a tracial weight. We then discuss Arveson's noncommutative Hardy space.

2.1. Weak $*$-topology. Let $\mathcal{M}$ be a von Neumann algebra with a predual $\mathcal{M}_{\sharp}$. Recall that the weak $*$-topology $\sigma\left(\mathcal{M}, \mathcal{M}_{\sharp}\right)$ on $\mathcal{M}$ is a topology on $\mathcal{M}$ induced from the predual space $\mathcal{M}_{\sharp}$. The following known result (for example, see Theorem 1.7.8 in [30]) is useful.

Lemma 2.1. Let $\mathcal{M}$ be a von Neumann algebra. If $\left\{e_{\lambda}\right\}_{\lambda \in \Lambda}$ is a net of projections in $\mathcal{M}$ such that $e_{\lambda} \rightarrow I$ in weak $*$-topology, then $e_{\lambda} x \rightarrow x, x e_{\lambda} \rightarrow x$ and $e_{\lambda} x e_{\lambda} \rightarrow x$ in weak $*$-topology for all $x$ in $\mathcal{M}$. 
2.2. Semifinite von Neumann Algebras. We begin with a description of a semifinite von Neumann algebra.

Let $\mathcal{M}$ be a von Neumann algebra, and let $\mathcal{M}^{+}$be the positive part of $\mathcal{M}$. Recall that a mapping $\tau: \mathcal{M}^{+} \rightarrow[0, \infty]$ is a tracial weight on $\mathcal{M}$ if

(1) $\tau(x+y)=\tau(x)+\tau(y)$ for $x, y \in \mathcal{M}^{+}$

(2) $\tau(a x)=a \tau(x)$ for $x \in \mathcal{M}^{+}$and $a \in[0, \infty]$

(3) $\tau\left(x x^{*}\right)=\tau\left(x^{*} x\right)$ for every $x \in \mathcal{M}$.

A tracial weight $\tau$ is called normal if $\tau: \mathcal{M}^{+} \rightarrow \mathbb{C}$ is continuous with respect to the weak *-topology. $\tau$ is faithful if for every $a \in \mathcal{M}^{+}, \tau\left(a^{*} a\right)=0$ implies $a=0 . \tau$ is said to be finite if $\tau(I)<\infty$, and semifinite if for any $x \in \mathcal{M}^{+}, x \neq 0$, there is a $y \in \mathcal{M}^{+}, y \neq 0$ such that $\tau(y)<\infty$ and $y \leq x$. A von Neumann algebra $\mathcal{M}$ is called semifinite if a faithful, normal semifinite $\tau$ exists.

The following lemma is well known.

LEMma 2.2. Let $\mathcal{M}$ be a von Neumann algebra with a semifinite, faithful, normal, tracial weight $\tau$. Then the following are true.

(1) There exists a family $\left\{e_{j}\right\}_{j \in J}$ of orthogonal projections in $\mathcal{M}$ such that (i) $\sum_{j} e_{j}$ converges to $I$ in weak $*$-topology and (ii) $\tau\left(e_{j}\right)<\infty$ for each $j \in J$.

(2) There exists a net $\left\{e_{\lambda}\right\}_{\lambda \in \Lambda}$ of projections in $\mathcal{M}$ such that (i) $e_{\lambda} \rightarrow I$ in weak $*$-topology and (ii) $\tau\left(e_{\lambda}\right)<\infty$ for each $\lambda \in \Lambda$.

Proof. It is not hard to see that (2) follows from (1). For the purpose of completeness, we sketch the proof of (1) here. Actually, we need only to show that every nonzero projection $e$ in $\mathcal{M}$ contains a nonzero subprojection $\tilde{e}$ such that $\tau(\tilde{e})<\infty$. Then the rest follows directly from Zorn's lemma.

Let $e$ be a nonzero projection in $\mathcal{M}$. Since $\tau$ is semifinite, then is a $y \in \mathcal{M}^{+}, y \neq 0$ such that $\tau(y)<\infty$ and $y \leq f$. Therefore, there exist a positive number $\lambda>0$ and a nonzero spectral projection $\tilde{e}$ of $y$ in $\mathcal{M}$ such that $\lambda \tilde{e} \leq y$. Hence $\tilde{e}$ is a non-zero subprojection of $e$ such that $\tau(\tilde{e})<\infty$.

2.3. $L^{p}$-spaces of semifinite von Neumann algebras. Let $\mathcal{M}$ be a von Neumann algebra with a semifinite, faithful, normal, tracial weight $\tau$. We let

$$
\mathcal{I}=\operatorname{span}\left\{M e M: e=e^{*}=e^{2} \in M \text { with } \tau(e)<\infty\right\}
$$

be the set of elementary operators in $\mathcal{M}$ (see Definition 3.1 in [33]). Then $\mathcal{I}$ is a two-sided ideal of $\mathcal{M}$.

For each $0<p<\infty$, we define a mapping $\|\cdot\|_{p}: \mathcal{I} \rightarrow[0, \infty)$ as follows

$$
\|x\|_{p}=\left(\tau(|x|)^{p}\right)^{\frac{1}{p}} \quad \text { for every } x \in \mathcal{I} .
$$

It is a highly trivial fact that $\|\cdot\|_{p}$ is a norm on $\mathcal{I}$ for $1 \leq p<\infty$, and a $p$-norm on $\mathcal{I}$ for $0<p<1$. (see Theorem 4.9 in [13]) 
Definition 2.3. Let $\mathcal{M}$ be a von Neumann algebra with a semifinite, faithful, normal, tracial weight $\tau$, and $\mathcal{I}=\operatorname{span}\left\{M e M: e=e^{*}=e^{2} \in M\right.$ with $\left.\tau(e)<\infty\right\}$ be the set of elementary operators in $\mathcal{M}$. We define $L^{p}(\mathcal{M}, \tau)$, for $0<p<\infty$, to be the completion of $\mathcal{I}$ under $\|\cdot\|_{p}$, i.e.

$$
L^{p}(\mathcal{M}, \tau)=\overline{\mathcal{I}}^{\|\cdot\|_{p}}
$$

As usual, we let $L^{\infty}(\mathcal{M}, \tau)$ be $\mathcal{M}$.

Notation 2.4. If $S$ is a subset of $L^{p}(\mathcal{M}, \tau)$ with $0<p<\infty$, we will denote by $[S]_{p}$ the closure of $S$ in $L^{p}(\mathcal{M}, \tau)$. If $S$ is a subset of $\mathcal{M}$, we will denote by $\bar{S}^{\text {w* }}$ the closure of $S$ in $\mathcal{M}$ under the weak *-topology.

The following two lemmas are well known.

Lemma 2.5. Let $\mathcal{M}$ be a von Neumann algebra with a semifinite, faithful, normal, tracial weight $\tau$. The following are true.

(1) (Hölder's Inequality) For $0<p, q, r \leq \infty$ with $1 / p+1 / q=1 / r$, we have

$$
\|x y\|_{r} \leq\|x\|_{p}\|y\|_{q} \quad \text { for all } x \in L^{p}(\mathcal{M}, \tau) \text { and } y \in L^{q}(\mathcal{M}, \tau) \text {. }
$$

(2) For each $0<r \leq \infty$, we have $\|a x b\|_{r} \leq\|a\|\|x\|_{r}\|b\|$ for $x \in L^{r}(\mathcal{M}, \tau)$ and $a, b \in \mathcal{M}$. Therefore, $L^{r}(\mathcal{M}, \tau)$ is an $\mathcal{M}$ bi-module for each $0<r \leq \infty$.

(3) (Duality) For any $1 \leq p<\infty$ and $1<q \leq \infty$ with $1 / p+1 / q=1$, we have

$$
\left(L^{p}(\mathcal{M}, \tau)\right)^{\sharp}=L^{q}(\mathcal{M}, \tau) \quad \text { (isometrically), }
$$

where the duality between $L^{p}(\mathcal{M}, \tau)$ and $L^{q}(\mathcal{M}, \tau)$ is given by $\langle x, y\rangle=\tau(x y)$. Thus, $L^{1}(\mathcal{M}, \tau)$ is the predual of $\mathcal{M}$.

ProOF. See [13].

Lemma 2.6. Let $\mathcal{M}$ be a von Neumann algebra with a semifinite, faithful, normal, tracial weight $\tau$ and $0<p<\infty$. If $\left\{e_{\lambda}\right\}_{\lambda \in \Lambda}$ is a net of projections in $\mathcal{M}$ such that such that $e_{\lambda} \rightarrow I$ in the weak $*$-topology, then for every $x \in L^{p}(\mathcal{M}, \tau)$

$$
\lim _{\lambda}\left\|e_{\lambda} x-x\right\|_{p}=0 ; \quad \lim _{\lambda}\left\|x e_{\lambda}-x\right\|_{p}=0 ; \quad \text { and } \quad \lim _{\lambda}\left\|e_{\lambda} x e_{\lambda}-x\right\|_{p}=0 .
$$

ProOf. For the purpose of completeness, we include a proof here. Notice that the set $\mathcal{I}$ of elementary operators of $\mathcal{M}$ is dense in $L^{p}(\mathcal{M}, \tau)$ (see Definition 2.3) and

$$
\left\|e_{\lambda} x e_{\lambda}-x\right\|_{p}=\left\|e_{\lambda}\left(x e_{\lambda}-x\right)+e_{\lambda} x-x\right\|_{p}, \quad \forall x \in L^{p}(\mathcal{M}, \tau) .
$$

Because of Lemma 2.5, it suffices to show that, for all $a, b \in \mathcal{M}$ and a projection $f$ in $\mathcal{M}$ with $\tau(f)<\infty$, we have $\lim _{\lambda}\left\|e_{\lambda}(a f b)-a f b\right\|_{p}=0$ and $\lim _{\lambda}\left\|(a f b) e_{\lambda}-a f b\right\|_{p}=0$. 
Assume that $0<p<2$. Let $q$ be a positive number such that $1 / p=1 / 2+1 / q$. We have

$$
\begin{aligned}
\left\|e_{\lambda}(a f b)-a f b\right\|_{p} & =\left\|\left(e_{\lambda}-I\right) a f b\right\|_{p} \\
& \leq\|b\|\left\|\left(e_{\lambda}-I\right) a f\right\|_{p} \\
& \leq\|b\|\left\|\left(e_{\lambda}-I\right) a f\right\|_{2}\|f\|_{q} \\
& =\|b\|\|f\|_{q}\left(\tau\left(f a^{*}\left(I-e_{\lambda}\right) a f\right)\right)^{1 / 2} \\
& =\|b\|\|f\|_{q}\left(\tau\left(\left(I-e_{\lambda}\right) a f a^{*}\right)\right)^{1 / 2} .
\end{aligned}
$$

(by Lemma 2.5)

( by Hölder's Inequality)

Observe that $e_{\lambda} \rightarrow I$ in weak $*$-topology and af $a^{*} \in L^{1}(\mathcal{M}, \tau)$ as $\tau(f)<\infty$. We have that

$$
\lim _{\lambda} \tau\left(\left(I-e_{\lambda}\right) a f a^{*}\right)=0 .
$$

It follows that $\lim _{\lambda}\left\|e_{\lambda}(a f b)-a f b\right\|_{p}=0$. Furthermore, we have that $\lim _{\lambda}\left\|(a f b) e_{\lambda}-a f b\right\|_{p}=$ $\lim _{\lambda}\left\|e_{\lambda} b^{*} f a^{*}-b^{*} f a^{*}\right\|_{p}=0$, for $0<p<2$.

Assume that $2 \leq p<\infty$. We have

$$
\begin{aligned}
\left\|e_{\lambda}(a f b)-a f b\right\|_{p} & \leq\|b\|\left\|\left(e_{\lambda}-I\right) a f\right\|_{p} \\
& =\|b\|\left(\tau\left(\left(f a^{*}\left(I-e_{\lambda}\right) a f\right)^{p / 2}\right)\right)^{1 / p} \\
& \left.\leq\|b\|\left\|\left(f a^{*}\left(I-e_{\lambda}\right) a f\right)^{\frac{p}{2}-1}\right\|^{1 / p}\left(\tau\left(f a^{*}\left(I-e_{\lambda}\right) a f\right)\right)^{1 / p} \quad \text { (by the property of } \tau\right)
\end{aligned}
$$

Note from Equation (2.1) that

$$
\lim _{\lambda} \tau\left(f a^{*}\left(I-e_{\lambda}\right) a f\right)=\lim _{\lambda} \tau\left(\left(I-e_{\lambda}\right) a f a^{*}\right)=0 .
$$

We have that $\lim _{\lambda}\left\|e_{\lambda}(a f b)-a f b\right\|_{p}=0$. Furthermore, we have that $\lim _{\lambda}\left\|(a f b) e_{\lambda}-a f b\right\|_{p}=$ $\lim _{\lambda}\left\|e_{\lambda} b^{*} f a^{*}-b^{*} f a^{*}\right\|_{p}=0$, for $2 \leq p<\infty$.

This ends the proof of the lemma.

2.4. Arveson's Non-Commutative Hardy Space. In this subsection, we will recall Arveson's definition of non-commutative Hardy spaces. Assume that $\mathcal{M}$ is a von Neumann algebra with a semifinite, faithful, normal tracial weight $\tau$. Assume $\mathcal{A} \subseteq \mathcal{M}$ is a weak*-closed subalgebra of $\mathcal{M}$, and let $\mathcal{D}=\mathcal{A} \cap \mathcal{A}^{*}$. Assume that $\Phi: \mathcal{M} \rightarrow \mathcal{D}$ is faithful, normal conditional expectation from $\mathcal{M}$ onto $\mathcal{D}$.

Definition 2.7. $\mathcal{A}$ is a called a semifinite subdigonal subalgebra, or a semifinite noncommutative Hardy space, with respect to $(\mathcal{M}, \Phi)$ if

(1) The restriction of $\tau$ on $\mathcal{D}=\mathcal{A} \cap \mathcal{A}^{*}$ is semifinite.

(2) $\Phi(x y)=\Phi(x) \Phi(y)$ for every $x, y \in \mathcal{A}$.

(3) $\mathcal{A}+\mathcal{A}^{*}$ is weak ${ }^{*}$ dense in $M$.

(4) $\tau(\Phi(x))=\tau(x)$ for every positive operator $x$ in $M$.

In this case, $\mathcal{A}$ will also be denoted by $H^{\infty}$. Furthermore, we denote $\left[\mathcal{A} \cap L^{p}(\mathcal{M}, \tau)\right]_{p}$ by $H^{p}$ for each $0<p<\infty$. 
REMARK 2.8. It was shown in [38] and [21] that such a subalgebra $H^{\infty}$ with respect to $(\mathcal{M}, \Phi)$ is maximal among semifinite subdigonal subalgebras satisfying (1), (2), (3) and (4). From this fact, it follows that

$$
H^{\infty}=\left\{a \in \mathcal{M}: \Phi(x a y)=0, \quad \forall x \in H^{\infty}, y \in H^{\infty} \cap \operatorname{Ker}(\Phi)\right\} .
$$

REMARK 2.9. Following notation from Definition 2.7, we know that the conditional expectation $\Phi: \mathcal{M} \rightarrow \mathcal{D}$ can be extended to a projection from $L^{p}(\mathcal{M}, \tau)$ onto $L^{p}(\mathcal{D}, \tau)$ for each $1 \leq p<\infty$ (see Proposition 2.3 in [38] or [5]). Such extended projection will still be denoted by $\Phi$. Moreover,

$$
\Phi(a x b)=a \Phi(x) b, \quad \forall a, b \in \mathcal{D}, x \in L^{p}(\mathcal{M}, \tau), 1 \leq p<\infty .
$$

Notation 2.10. We will let $H_{0}^{\infty}=\operatorname{ker}(\Phi) \cap H^{\infty}$ and $H_{0}^{p}=\operatorname{ker}(\Phi) \cap H^{p}$.

The next result follows directly from Definition 2.7 and can be found in Lemma 3.1 of [5].

LEMma 2.11. If e is a projection in $\mathcal{D}=H^{\infty} \cap\left(H^{\infty}\right)^{*}$ with $0<\tau(e)<\infty$, then e $H^{\infty} e$ is a finite subdiagonal subalgebra of e $\mathcal{M} e$, and $\left[e H^{\infty} e\right]_{p}=e H^{p} e$.

We will need the following technical lemma in the later sections.

Lemma 2.12. Suppose $\mathcal{M}$ is a von Neumann algebra with a semifinite, faithful, normal, tracial weight $\tau$. Let $H^{\infty}$ be a semifinite subdiagonal subalgebra in $\mathcal{M}$ in the sense of Definition 2.7 (namely, the restriction of $\tau$ on $\mathcal{D}=H^{\infty} \cap\left(H^{\infty}\right)^{*}$ is semifinite).

Then for every $x \in L^{p}(M, \tau)$ with $0<p<\infty$ and for every $e \in \mathcal{D}$ with $0<\tau(e)<\infty$, there exist an $h_{1}, h_{3} \in e H^{\infty} e$ and an $h_{2}, h_{4} \in e H^{p} e$ such that:

(i) $h_{1} h_{2}=e=h_{2} h_{1}$ and $h_{3} h_{4}=h_{4} h_{3}=e$

(ii) $h_{1}$ ex and $x e h_{3}$ are in $\mathcal{M}$.

Proof. Let

$$
e x=\sqrt{e x x^{*} e} u_{1}=\left|x^{*} e\right| u_{1}
$$

be the polar decomposition of $e x$ in $L^{p}(\mathcal{M}, \tau)$, where $u_{1}$ is a partial isometry in $\mathcal{M}$ and $\left|x^{*} e\right|$ is a positive operator in $L^{p}(\mathcal{M}, \tau)$. It is not hard to see that $\left|x^{*} e\right| \in e L^{p}(\mathcal{M}, \tau) e=L^{p}(e \mathcal{M} e, \tau)$. Since $0<\tau(e)<\infty$, we know that $e \mathcal{M} e$ is a finite von Neumann algebra with a faithful normal tracial state $\frac{1}{\tau(e)} \tau$. And, from Lemma 2.11, it follows that $e H^{\infty} e$ is a finite subdigonal subalgebra of $e \mathcal{M} e$ with $\left[e H^{\infty} e\right]_{p}=e H^{p} e$. Note that $\left|x^{*} e\right| \in L^{p}(e \mathcal{M} e, \tau)$ and $0<\tau(e)<\infty$. Then $w=\left(e+\left|x^{*} e\right|\right)^{-1}$ is an invertible operator in $e \mathcal{M} e$ with $w^{-1} \in L^{p}\left(e \mathcal{M} e, \frac{1}{\tau(e)} \tau\right)$. From Theorem 3.1 in [3], there exist a unitary $v$ in $e \mathcal{M} e$, an $h_{1} \in e H^{\infty} e$ and an $h_{2} \in e H^{p} e$ such that (i) $h_{1} h_{2}=e=h_{2} h_{1}$, and $\left(\mathrm{ii}_{a}\right) w=v h_{1}$. Now from ( $\left.\mathrm{ii}_{a}\right)$ we have $\left(\mathrm{ii}_{b}\right) h_{1}\left|x^{*} e\right|=v^{*} w\left|x^{*} e\right|=$ $v^{*}\left(e+\left|x^{*} e\right|\right)^{-1}\left|x^{*} e\right| \in e \mathcal{M} e \subseteq \mathcal{M}$. Hence, from (ii $b$ ) and the fact that $u_{1}$ is a partial isometry in $\mathcal{M}$, we obtain that (ii) $h_{1} e x=h_{1}\left|x^{*} e\right| u_{1} \in \mathcal{M}$. The proof for the existence of $h_{3}$ and $h_{4}$ is similar. This ends the proof of the lemma.

The following lemma is also useful. 
Lemma 2.13. Suppose $\mathcal{M}$ is a von Neumann algebra with a semifinite, faithful, normal, tracial weight $\tau$. Let $H^{\infty}$ be a semifinite subdiagonal subalgebra with respect to $(\mathcal{M}, \Phi)$, where $\Phi$ is a faithful, normal conditional expectation from $\mathcal{M}$ onto $\mathcal{D}=H^{\infty} \cap\left(H^{\infty}\right)^{*}$.

Then there exists a net $\left\{e_{\lambda}\right\}_{\lambda \in \Lambda}$ of projections in $\mathcal{D}$ such that such that

(i) $e_{\lambda} \rightarrow I$ in the weak $*$-topology of $\mathcal{M}$ and $\tau\left(e_{\lambda}\right)<\infty$ for each $\lambda \in \Lambda$.

(ii) We have, for every $x \in L^{p}(\mathcal{M}, \tau)$ with $0<p<\infty$,

$$
\lim _{\lambda}\left\|e_{\lambda} x-x\right\|_{p}=0 ; \quad \lim _{\lambda}\left\|x e_{\lambda}-x\right\|_{p}=0 ; \quad \text { and } \quad \lim _{\lambda}\left\|e_{\lambda} x e_{\lambda}-x\right\|_{p}=0 .
$$

Proof. Since $H^{\infty}$ is a semifinite subdiagonal subalgebra of $\mathcal{M}$, the restriction of $\tau$ on $\mathcal{D}$ is semifinite. By Lemma 2.2, there exists a net $\left\{e_{\lambda}\right\}_{\lambda \in \Lambda}$ of projections in $\mathcal{D}$ such that $e_{\lambda} \rightarrow I$ in the weak $*$-topology of $\mathcal{D}$ and $\tau\left(e_{\lambda}\right)<\infty$ for each $\lambda \in \Lambda$. Thus

$$
\lim _{\lambda}\left|\tau\left(e_{\lambda} z-z\right)\right|=0, \quad \forall z \in L^{1}(\mathcal{D}, \tau) .
$$

For each $y \in L^{1}(\mathcal{M}, \tau)$, we have

$$
\lim _{\lambda}\left|\tau\left(e_{\lambda} y-y\right)\right|=\lim _{\lambda}\left|\tau\left(\Phi\left(e_{\lambda} y-y\right)\right)\right|=\lim _{\lambda}\left|\tau\left(e_{\lambda} \Phi(y)-\Phi(y)\right)\right|=0 .
$$

i.e.

(i) $e_{\lambda} \rightarrow I$ in the weak $*$-topology of $\mathcal{M}$ and $\tau\left(e_{\lambda}\right)<\infty$ for each $\lambda \in \Lambda$.

From (i) and Lemma 2.6, we induce that

(ii) For every $x \in L^{p}(\mathcal{M}, \tau)$,

$$
\lim _{\lambda}\left\|e_{\lambda} x-x\right\|_{p}=0 ; \quad \lim _{\lambda}\left\|x e_{\lambda}-x\right\|_{p}=0 ; \quad \text { and } \quad \lim _{\lambda}\left\|e_{\lambda} x e_{\lambda}-x\right\|_{p}=0 .
$$

This ends the proof of the lemma.

Now we recall the following definition for the row sum of subspaces in $L^{p}(\mathcal{M}, \tau)$ for $0<p \leq \infty$ as follows.

Definition 2.14. Let $\mathcal{M}$ be a von Neumann algebra with a semifinite, normal faithful, tracial weight $\tau$ and $0<p<\infty$. Let $X$ be a closed subspace of $L^{p}(\mathcal{M}, \tau)$. Then $X$ is called an internal row sum of closed subspaces $\left\{X_{i}\right\}_{i \in \mathcal{I}}$ of $L^{p}(\mathcal{M}, \tau)$, denoted by $X=\bigoplus_{i \in \mathcal{I}}^{\text {row }} X_{i}$, if

(1) $X_{j} X_{i}^{*}=\{0\}$ for all distinct $i, j \in \mathcal{I}$; and

(2) the linear span of $\left\{X_{i}: i \in \mathcal{I}\right\}$ is dense in $X$, i.e. $X=\left[\operatorname{span}\left\{X_{i}: i \in \mathcal{I}\right\}\right]_{p}$.

Definition 2.15. Let $\mathcal{M}$ be a von Neumann algebra. Let $X$ be a weak $*$-closed subspace of $\mathcal{M}$. Then $X$ is called an internal row sum of a family of weak ${ }^{*}$-closed subspaces $\left\{X_{i}\right\}_{i \in \mathcal{I}}$ of $\mathcal{M}$, denoted by $X=\bigoplus_{i \in \mathcal{I}}^{\text {row }} X_{i}$, if

(1) $X_{j} X_{i}^{*}=\{0\}$ for all distinct $i, j \in \mathcal{I}$; and

(2) the linear span of $\left\{X_{i}: i \in \mathcal{I}\right\}$ is weak*-dense in $X$, i.e. $X={\overline{\operatorname{span}\left\{X_{i}: i \in \mathcal{I}\right\}}}^{w *}$. 


\section{Beurling-Blecher-Labuschagne Theorem for Semifinite Hardy Spaces, $\mathrm{p}=2$}

3.1. Main Result. In this section, we will prove a Beurling-Blecher-Labuschagne type theorem for semifinite non-commutative Hardy spaces.

TheOREm 3.1. Let $\mathcal{M}$ be a von Neumann algebra with a faithful, normal, semifinite tracial weight $\tau$, and $H^{\infty}$ be a weak $k^{*}$-closed subalgebra of $\mathcal{M}$. Let $\mathcal{D}=H^{\infty} \cap\left(H^{\infty}\right)^{*}$ be a von Neumann subalgebra of $\mathcal{M}$, and $\Phi: \mathcal{M} \rightarrow \mathcal{D}$ be a faithful normal condition expectation.

Assume that $H^{\infty}$ is a semifinite subdigonal subalgebra with respect to $(\mathcal{M}, \Phi)$ (see Definition 2.7). Let $\mathcal{K}$ be a closed subspace of $L^{2}(\mathcal{M}, \tau)$ satisfying $H^{\infty} \mathcal{K} \subseteq \mathcal{K}$. Then there exist a closed subspace $Y$ of $L^{2}(\mathcal{M}, \tau)$ and a family $\left\{u_{\lambda}\right\}_{\lambda \in \Lambda}$ of partial isometries in $\mathcal{M}$, satisfying

(i) $u_{\lambda} Y^{*}=0$ for all $\lambda \in \Lambda$.

(ii) $u_{\lambda} u_{\lambda}^{*} \in \mathcal{D}$ and $u_{\lambda} u_{\mu}^{*}=0$ for all $\lambda, \mu \in \Lambda$ with $\lambda \neq \mu$.

(iii) $Y=\left[H_{0}^{\infty} Y\right]_{2}$, where $H_{0}^{\infty}=H^{\infty} \cap \operatorname{ker}(\Phi)$.

(iv) $\mathcal{K}=Y \oplus\left(\oplus_{\lambda \in \Lambda} H^{2} u_{\lambda}\right)$

The proof of this result uses a similar idea as the one in [7] for finite von Neumann algebras. We will modify the argument in [7] to prove preceding result for the case of semifinite von Neumann algebras. First, we present a series of technical lemmas.

3.2. Some lemmas. Following the notation above, we let $\mathcal{M}$ be a von Neumann algebra with a faithful, normal, semifinite tracial weight $\tau$ and $H^{\infty}$ be a semifinite subdigonal subalgebra of $\mathcal{M}$. Let $\mathcal{D}=H^{\infty} \cap\left(H^{\infty}\right)^{*}$ be a von Neumann subalgebra of $\mathcal{M}$ and $\Phi: \mathcal{M} \rightarrow \mathcal{D}$ a faithful normal conditional expectation. From Remark 2.9, we know that $\Phi$ can be extended to a positive contraction from $L^{p}(\mathcal{M}, \tau)$ onto $L^{p}(\mathcal{D}, \tau)$ for each $1 \leq p<\infty$ such that

$$
\Phi(a x b)=a \Phi(x) b, \quad \forall a, b \in \mathcal{D}, x \in L^{p}(\mathcal{M}, \tau), 1 \leq p<\infty .
$$

We find the following lemma is useful.

Lemma 3.2. Let $\mathcal{M}$ be a von Neumann algebra with a faithful, normal, semifinite tracial weight $\tau$, and $H^{\infty}$ be a semifinite subdigonal subalgebra of $\mathcal{M}$. If $x$ in $L^{1}(\mathcal{M}, \tau)$ satisfies

$$
\tau(x z)=0 \quad \text { for all } z \in H^{\infty}+\left(H^{\infty}\right)^{*},
$$

then $x=0$.

Proof. Assume that $x$ in $L^{1}(\mathcal{M}, \tau)$ satisfies

$$
\tau(x z)=0 \quad \text { for all } z \in H^{\infty}+\left(H^{\infty}\right)^{*} .
$$

Since $H^{\infty}$ is a semifinite subdigonal subalgebra of $\mathcal{M}, H^{\infty}+\left(H^{\infty}\right)^{*}$ is weak ${ }^{*}$-dense in $\mathcal{M}$. From the fact that $x \in L^{1}(\mathcal{M}, \tau)$, we get that

$$
\tau(x z)=0 \quad \text { for all } z \in \mathcal{M} .
$$

As $L^{1}(\mathcal{M}, \tau)$ is the predual space of $\mathcal{M}$, we must have $x=0$. 
Lemma 3.3. Let $\mathcal{K}$ be a closed subspace of $L^{2}(\mathcal{M}, \tau)$ satisfying $H^{\infty} \mathcal{K} \subseteq \mathcal{K}$. Let

$$
X=\mathcal{K} \ominus\left[H_{0}^{\infty} \mathcal{K}\right]_{2} \subseteq \mathcal{K} \subseteq L^{2}(\mathcal{M}, \tau)
$$

Then the following are true.

(i) $X X^{*} \subseteq L^{1}(\mathcal{D}, \tau)$.

(ii) $X$ is a left $\mathcal{D}$-module, i.e. for every $d \in \mathcal{D}$ and $x \in X$, we have $d x \in X$.

(iii) Let $x$ be an element in $X$ and $x=h u$ be the polar decomposition of $x$ in $L^{2}(\mathcal{M}, \tau)$, where $u$ is a partial isometry in $\mathcal{M}$ and $h=\left|x^{*}\right| \in L^{2}(\mathcal{M}, \tau)$. Then

(a) $h \in L^{2}(\mathcal{D}, \tau)$ and $u u^{*} \in \mathcal{D}$;

(b) $[\mathcal{D} x]_{2}=L^{2}(\mathcal{D}, \tau) u$;

(c) $\left[H^{\infty} x\right]_{2}=H^{2} u$. In particular, $H^{2} u \subseteq X$.

(iv) There exists a family $\left\{u_{\lambda}\right\}_{\lambda \in \Lambda}$ of partial isometries in $\mathcal{M}$ such that

(a) $X=\oplus_{\lambda \in \Lambda} H^{2} u_{\lambda}$;

(b) $u_{\lambda} u_{\lambda}^{*}$ is a projection in $\mathcal{D}$; and

(c) $u_{\lambda} u_{\mu}^{*}=0$ for all $\lambda, \mu \in \Lambda$ with $\lambda \neq \mu$.

Proof. (i): It is equivalent to show that for every $x, y \in X, y x^{*} \in L^{1}(\mathcal{D}, \tau)$.

Assume that $x, y \in X \subseteq L^{2}(\mathcal{M}, \tau)$. Thus $y x^{*} \in L^{1}(\mathcal{M}, \tau)$. Recall $\Phi: L^{1}(\mathcal{M}, \tau) \rightarrow L^{1}(\mathcal{D}, \tau)$ is a positive contraction such that

$$
\Phi\left(d_{1} a d_{2}\right)=d_{1} \Phi(a) d_{2}, \quad \forall d_{1}, d_{2} \in \mathcal{D} \text { and } a \in L^{1}(\mathcal{M}, \tau),
$$

and thus

$$
\Phi(d a)=d \Phi(a), \quad \forall d \in L^{1}(\mathcal{D}, \tau) \text { and } a \in \mathcal{M}
$$

Thus, to prove that $y x^{*} \in L^{1}(\mathcal{D}, \tau)$, it is enough to show that $y x^{*}-\Phi\left(y x^{*}\right)=0$. By Lemma 3.2 and the fact that $y x^{*}-\Phi\left(y x^{*}\right) \in L^{1}(\mathcal{M}, \tau)$, we need only to prove that

$$
\tau\left(\left[y x^{*}-\Phi\left(y x^{*}\right)\right] z\right)=0 \quad \text { for every } z \in H^{\infty}+\left(H^{\infty}\right)^{*} .
$$

We will proceed the proof according to the cases $(1) z \in H_{0}^{\infty},(2) z \in \mathcal{D}$, and $(3) z \in\left(H_{0}^{\infty}\right)^{*}$. Case (1): Let $z \in H_{0}^{\infty}$. Then

$$
\begin{array}{rlrl}
\tau\left(\left[y x^{*}-\Phi\left(y x^{*}\right)\right] z\right) & =\tau\left(y x^{*} z\right)-\tau\left(\Phi\left(y x^{*}\right) z\right) & \\
& =\tau\left(y x^{*} z\right)-\tau\left(\Phi\left(\Phi\left(y x^{*}\right) z\right)\right) & \\
& =\tau\left(z y x^{*}\right)-\tau\left(\Phi\left(y x^{*}\right) \Phi(z)\right) & & (\Phi \text { is trace preserving) } \\
& =0 & \left(\text { as } x, y \text { are in } X \text { and } z \text { is in } H_{0}^{\infty}\right)
\end{array}
$$

Case (2): Let $z \in \mathcal{D}$. Then

$$
\begin{array}{rlrl}
\tau\left(\left[y x^{*}-\Phi\left(y x^{*}\right)\right] z\right) & =\tau\left(\Phi\left(\left[y x^{*}-\Phi\left(y x^{*}\right)\right] z\right)\right) & \quad \text { ( } \Phi \text { is trace preserving) } \\
& =\tau\left(\left[\Phi\left(y x^{*}\right)-\Phi\left(y x^{*}\right)\right] z\right) \\
& =0 . & \quad\left(\text { as } x, y \text { are in } X \text { and } z \text { is in } H_{0}^{\infty}\right)
\end{array}
$$


Case (3): Let $z \in\left(H_{0}^{\infty}\right)^{*}$. Then

$$
\begin{array}{rlr}
\tau\left(\left[\left(y x^{*}\right)-\Phi\left(y x^{*}\right)\right] z\right) & =\tau\left(y x^{*} z\right)-\tau\left(\Phi\left(y x^{*}\right) z\right) & \\
& =\tau\left(y\left(z^{*} x\right)^{*}\right)-\tau\left(\Phi\left(\Phi\left(y x^{*}\right) z\right)\right) & (\Phi \text { is trace preserving) } \\
& =\tau\left(y\left(z^{*} x\right)^{*}\right)-\tau\left(\Phi\left(y x^{*}\right) \Phi(z)\right) & (\text { by equation 3.1) } \\
& =0 & \left(\text { as } x, y \text { are in } X \text { and } z \text { is in } H_{0}^{\infty}\right)
\end{array}
$$

This ends the proof of part (i).

(ii): Let $d \in \mathcal{D}$ and $x \in X \subseteq \mathcal{K}$. Since $H^{\infty} \mathcal{K} \subseteq \mathcal{K}$, we have $d x \in \mathcal{K}$. Now, for $h_{0} \in H_{0}^{\infty}$ and $k \in K$,

$$
\tau\left(h_{0} k(d x)^{*}\right)=\tau\left(h_{0} k x^{*} d^{*}\right)=\tau\left(d^{*} h_{0} k x^{*}\right)=0,
$$

as $d^{*} h_{0} \in H_{0}^{\infty}$, and $x \in X=\mathcal{K} \ominus\left[H_{0}^{\infty} \mathcal{K}\right]_{2}$. Hence $d x \perp\left[H_{0}^{\infty} K\right]_{2}$. Thus $d x \in X$ and $X$ is a left $\mathcal{D}$-module.

(iii): Assume $x$ is an element in $X$. Let $x=h u$ be the polar decomposition of $x$ in $L^{2}(\mathcal{M}, \tau)$, where $u$ is a partial isometry in $\mathcal{M}$ and $h=\left|x^{*}\right| \in L^{2}(\mathcal{M}, \tau)$. From the result in (i), we know that $h$ is in $L^{2}(\mathcal{D}, \tau)$. Therefore $u u^{*}$, as the range projection of $h$, is in $\mathcal{D}$. This shows that (a) is true.

From (a), it follows that $\left[L^{2}(\mathcal{D}, \tau) u u^{*}\right]_{2}=L^{2}(\mathcal{D}, \tau)\left(u u^{*}\right)$. Observe that $u u^{*}$ is the range projection of $h$. Therefore, we have $[\mathcal{D} h]_{2}=L^{2}(\mathcal{D}, \tau)\left(u u^{*}\right)$, whence

$$
[\mathcal{D} h]_{2} u=L^{2}(\mathcal{D}, \tau)\left(u u^{*} u\right)=L^{2}(\mathcal{D}, \tau) u \text {. }
$$

We claim that

$$
[\mathcal{D} x]_{2}=[\mathcal{D} h]_{2} u .
$$

In fact, let $\xi \in[\mathcal{D} h]_{2}$. There exists a sequence $\left\{\xi_{n}\right\}_{n \in \mathbb{N}}$ in $\mathcal{D} h$ such that $\xi_{n} \rightarrow \xi$ in $\|\cdot\|_{2}$-norm. Then we have that $\xi_{n} u \rightarrow \xi u$ in $\|\cdot\|_{2}$-norm. From the fact that $\xi_{n} u \in[\mathcal{D} h u]_{2}$, we conclude that $\xi u \in[\mathcal{D} h u]_{2}$. Therefore, we have that

$$
[\mathcal{D} h]_{2} u \subseteq[\mathcal{D h u}]_{2}=[\mathcal{D} x]_{2}
$$

Now let $\xi \in[\mathcal{D} h u]_{2}=[\mathcal{D} x]_{2}$. There exists a sequence $\left\{d_{n}\right\}_{n \in \mathbb{N}}$ in $\mathcal{D}$ such that $d_{n} h u \rightarrow \xi$ in $\|\cdot\|_{2^{-n o r m}}$ Let $\eta_{n}=d_{n} h u u^{*}=d_{n} h \in \mathcal{D} h$. Then $\eta_{n} \rightarrow \xi u^{*} \in[\mathcal{D} h]_{2}$ in $\|\cdot\|_{2}$-norm. Thus $\eta_{n} u=d_{n} h u \rightarrow \xi u^{*} u$ in $\|\cdot\|_{2}$-norm. Combining with the fact that $d_{n} h u \rightarrow \xi$, we get $\xi=\left(\left(\xi u^{*}\right) u\right) \in[\mathcal{D} h]_{2} u$. Or

$$
[\mathcal{D h u}]_{2}=[\mathcal{D} x]_{2} \subseteq[\mathcal{D} h]_{2} u .
$$

From equation (3.3), equation (3.4) and equation (3.2), we conclude that

$$
[\mathcal{D} x]_{2}=[\mathcal{D} h]_{2} u=L^{2}(\mathcal{D}, \tau) u \text {. }
$$

This ends the proof of part (b). The proof of (c) is similar to (b).

(iv) We may assume that $X \neq 0$. From the result in (iii) and Zorn's lemma, we may assume that there exists a maximal family $\left\{u_{\lambda}\right\}_{\lambda \in \Lambda}$ of nonzero partial isometries in $\mathcal{M}$ with respect to

(a) $H^{2} u_{\lambda} \subseteq X$ for each $\lambda \in \Lambda$;

(b) $u_{\lambda} u_{\lambda}^{*}$ is a projection in $\mathcal{D}$; and 
(c) $u_{\lambda} u_{\mu}^{*}=0$ for all $\lambda, \mu \in \Lambda$ with $\lambda \neq \mu$.

We will show that

(a) $X=\oplus_{\lambda \in \Lambda} H^{2} u_{\lambda}$.

In fact, from $\left(\mathrm{a}_{1}\right)$, we know that each $H^{2} u_{\lambda} \subseteq X$. Combining with (c), we conclude that $\left\{H^{2} u_{\lambda}\right\}_{\lambda \in \Lambda}$ is a family of orthogonal subspaces of $X$, whence $\oplus_{\lambda \in \Lambda} H^{2} u_{\lambda}$ is a subspace of $X$.

Now assume that $X \ominus\left(\oplus_{\lambda \in \Lambda} H^{2} u_{\lambda}\right)$ is not equal to 0 . Pick a nonzero $x$ in $X \ominus\left(\oplus_{\lambda \in \Lambda} H^{2} u_{\lambda}\right)$ and assume that $x=h u$ is the polar decomposition of $x$ in $L^{2}(\mathcal{M}, \tau)$, where $u$ is a nonzero partial isometry in $\mathcal{M}$ and $h=\left|x^{*}\right| \in L^{2}(\mathcal{M}, \tau)$. It follows from the result proved in (iii) that $H^{2} u \subseteq X$ and $u u^{*}$ is in $\mathcal{D}$.

By Lemma 2.13, there exists a net $\left\{e_{j}\right\}_{j \in J}$ of projections in $\mathcal{D}$ such that such that $e_{j} \rightarrow I$ in the weak $*$-topology and $\tau\left(e_{j}\right)<\infty$ for each $j \in J$.

Let $j \in J$. Then by the choice of $x$, we get that $H^{2} u_{\lambda}$ and $x$ are orthogonal. So,

$$
\tau\left(d e_{j} u_{\lambda} x^{*}\right)=0, \quad \forall d \in \mathcal{D} .
$$

From (i), $e_{j} u_{\lambda} x^{*}$ is in $L^{1}(\mathcal{D}, \tau)$. By Lemma 3.2 we conclude that $e_{j} u_{\lambda} x^{*}=0$ for each $j \in J$.

As $u_{\lambda} x^{*} \in L^{2}(\mathcal{M}, \tau), \lim _{j}\left\|e_{j} u_{\lambda} x^{*}-u_{\lambda} x^{*}\right\|_{2}=0$ by Lemma 2.6. Thus we have that $u_{\lambda} x^{*}=$ $u_{\lambda} u^{*} h=0$. The fact that the initial projection of $u^{*}$ is the range projection of $h$ induces that $u_{\lambda} u^{*}=0$. Therefore, $u$ is a nonzero partial isometry in $\mathcal{M}$ such that $H^{2} u \subseteq X, u u^{*} \in \mathcal{D}$, and $u_{\lambda} u^{*}=0$ for each $\lambda \in \Lambda$. This contradicts the assumption that the family $\left\{u_{\lambda}\right\}_{\lambda \in \Lambda}$ is maximal with respect to $\left(\mathrm{a}_{1}\right)$, (b) and (c). Therefore, $X=\oplus_{\lambda \in \Lambda} H^{2} u_{\lambda}$. This concludes the proof of part (iv).

LEMma 3.4. Let $\mathcal{K}$ be a closed subspace of $L^{2}(\mathcal{M}, \tau)$ satisfying $H^{\infty} \mathcal{K} \subseteq \mathcal{K}$. Let

$$
X=\mathcal{K} \ominus\left[H_{0}^{\infty} \mathcal{K}\right]_{2} \text { and } Y=\mathcal{K} \ominus\left[H^{\infty} X\right]_{2} \text {. }
$$

Then the following are true.

(i) $Y X^{*}=0$, or equivalently $X Y^{*}=0$.

(ii) $Y=\left[H_{0}^{\infty} Y\right]_{2}$

Proof. (i) We will show that $y x^{*}=0$ for every $y \in Y$ and $x \in X$.

Note that $Y \subseteq K \subseteq L^{2}(\mathcal{M}, \tau)$ and $X \subseteq K \subseteq L^{2}(\mathcal{M}, \tau)$. We have that $Y X^{*} \subseteq L^{1}(\mathcal{M}, \tau)$. Assume $y \in Y$ and $x \in X$. Then by Lemma [3.2, it suffices to show that

$$
\tau\left(y x^{*} z\right)=0 \quad \text { for every } z \in H^{\infty}+\left(H^{\infty}\right)^{*} .
$$

We will proceed the proof according to the cases $(1) z \in H_{0}^{\infty},(2) z \in \mathcal{D}$, and $(3) z \in\left(H_{0}^{\infty}\right)^{*}$. Case (1): Let $z \in H_{0}^{\infty}$. Then

$$
\tau\left(y x^{*} z\right)=\tau\left(z y x^{*}\right)=0,
$$

since $x \in X, z y \in H_{0}^{\infty} K$, and $X \perp\left[H_{0}^{\infty} K\right]_{2}$.

Case (2): Let $z \in \mathcal{D}$. Then

$$
\tau\left(y x^{*} z\right)=\tau\left(y\left(z^{*} x\right)^{*}\right)=0,
$$

as $y \in Y, z^{*} x \in H^{\infty} X$, and $Y \perp H^{\infty} X$. 
Case (3): Let $z \in\left(H_{0}^{\infty}\right)^{*}$. Then

$$
\tau\left(y x^{*} z\right)=\tau\left(y\left(z^{*} x\right)^{*}\right)=0
$$

as $y \in Y$, and $z^{*} x \in H_{0}^{\infty} X$.

Therefore, $Y X^{*}=0$, which ends the proof of (i).

(ii) From part (i), we know that $Y X^{*}=0$, whence $H_{0}^{\infty} Y X^{*}=0$. Recall $Y=\mathcal{K} \ominus\left[H^{\infty} X\right]_{2}$. It follows that $\left[H_{0}^{\infty} Y\right]_{2} \subseteq Y$. Let $Z=Y \ominus\left[H_{0}^{\infty} Y\right]_{2}=0$. To prove (ii), it suffices to show that $Z Z^{*}=0$. Because $Z \subseteq Y$, we have that $Z \perp\left[H^{\infty} X\right]_{2}$, whence $Z \perp\left[H_{0}^{\infty}\left(Y \oplus\left[H^{\infty} X\right]_{2}\right)\right]_{2}$. This implies that $Z \perp\left[H_{0}^{\infty} \mathcal{K}\right]_{2}$. Note that $X=\mathcal{K} \ominus\left[H_{0}^{\infty} \mathcal{K}\right]_{2}$. We conclude that $Z \subseteq X$. Note that $Y X^{*}=0$. Since $Z \subseteq X$ and $Z \subseteq Y$, we have that $Z Z^{*} \subseteq Y X^{*}=0$. This ends the proof of (ii).

3.3. Proof of Theroem 3.1. We are ready to prove the main result in this section.

ProOF. Recall that $\mathcal{K}$ is a closed subspace of $L^{2}(\mathcal{M}, \tau)$ satisfying $H^{\infty} \mathcal{K} \subseteq \mathcal{K}$. Let

$$
X=\mathcal{K} \ominus\left[H_{0}^{\infty} \mathcal{K}\right]_{2} \text { and } Y=\mathcal{K} \ominus\left[H^{\infty} X\right]_{2} .
$$

By Lemma 3.3, there exists a family $\left\{u_{\lambda}\right\}_{\lambda \in \Lambda}$ of partial isometries in $\mathcal{M}$ such that

$$
X=\oplus_{\lambda \in \Lambda} H^{2} u_{\lambda}
$$

and

$$
u_{\lambda} u_{\lambda}^{*} \text { is a projection in } \mathcal{D} \text {, and } u_{\lambda} u_{\mu}^{*}=0 \text { for all } \lambda, \mu \in \Lambda \text { with } \lambda \neq \mu \text {. }
$$

By the choice of $Y$, we have

$$
\mathcal{K}=Y \oplus X=Y \oplus\left(\oplus_{\lambda \in \Lambda} H^{2} u_{\lambda}\right) .
$$

Moreover, from Lemma 3.4, we know that

$$
u_{\lambda} Y^{*}=0 \quad \text { for all } \lambda \in \Lambda \text {; }
$$

and

$$
Y=\left[H_{0}^{\infty} Y\right]_{2}
$$

This ends the proof of Theorem 3.1 .

\section{Beurling-Blecher-Labuschagne Theorem for Semifinite Hardy Spaces, $1 \leq p \leq \infty$}

\subsection{Dense subspaces.}

Proposition 4.1. Let $\mathcal{M}$ be a von Neumann algebra with a faithful, normal, semifinite tracial weight $\tau$, and $H^{\infty}$ be a semifinite subdigonal subalgebra of $\mathcal{M}$. Let $1 \leq p<\infty$. Assume that $\mathcal{K}$ is a closed subspace in $L^{p}(\mathcal{M}, \tau)$ such that $H^{\infty} \mathcal{K} \subseteq \mathcal{K}$. Then the following statements are true.

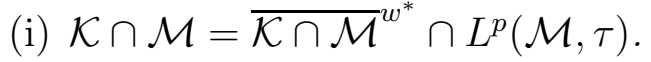

(ii) $\mathcal{K}=[\mathcal{K} \cap \mathcal{M}]_{p}$. 
ProOF. (i) It is easily observed that

$$
\mathcal{K} \cap \mathcal{M} \subseteq \overline{\mathcal{K} \cap \mathcal{M}}^{w^{*}} \cap L^{p}(\mathcal{M}, \tau) .
$$

We will show that

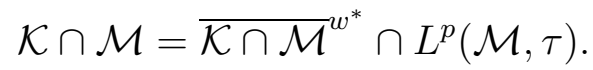

Assume, to the contrary, that $\mathcal{K} \cap \mathcal{M} \varsubsetneqq{\overline{\mathcal{K} \cap \mathcal{M}^{w}}}^{w^{*}} \cap L^{p}(\mathcal{M}, \tau)$. Then there exists an $x \in$ $\bar{K}_{1} \cap \mathcal{M}^{w^{*}} \cap L^{p}(\mathcal{M}, \tau)$ such that $x \notin \mathcal{K} \cap \mathcal{M}$. Then by the Hahn-Banach theorem, there exists $\varphi \in L^{p}(\mathcal{M}, \tau)^{\#}=L^{q}(\mathcal{M}, \tau)$ (where $\frac{1}{p}+\frac{1}{q}=1$ ) such that $\varphi(x) \neq 0$ and $\varphi(y)=0$ for every $y \in \mathcal{K} \cap \mathcal{M}$. Equivalently, there exists a $\xi \in L^{q}(\mathcal{M}, \tau)$ such that $\tau(x \xi) \neq 0$ and $\tau(y \xi)=0$ for every $y \in \mathcal{K} \cap \mathcal{M}$.

By Lemma 2.13, there exists a net $\left\{e_{\lambda}\right\}_{\lambda \in \Lambda}$ of projections in $\mathcal{D}$ such that $\tau\left(e_{\lambda}\right)<\infty$ for each $\lambda \in \Lambda$, and $\lim _{\lambda} \tau\left(e_{\lambda} x \xi\right)=\tau(x \xi)$. So, we can always assume that there exists a projection $e$ in $\mathcal{D}$ with $0<\tau(e)<\infty$ such that $\tau(e x \xi) \neq 0$ and $\tau(e y \xi)=0$ for every $y \in \mathcal{K}$ (as $\mathcal{K}$ is $H^{\infty}$-invariant and $\left.e \in \mathcal{D} \subseteq H^{\infty}\right)$.

Now we claim that $\xi e \in L^{1}(\mathcal{M}, \tau)$, as $\|\xi e\|_{1} \leq\|\xi\|_{q}\|e\|_{p}<\infty$.

Since $x \in \bar{K}_{1} \cap \mathcal{M}^{w^{*}} \cap L^{p}(\mathcal{M}, \tau)$, we can find a net $\left\{y_{i}\right\}_{i \in I}$ in $\mathcal{K} \cap \mathcal{M}$, such that $y_{i} \rightarrow x$ in the weak*-topology. Combining this with the fact that $\xi e \in L^{1}(\mathcal{M}, \tau)$, we have

$$
\tau(e x \xi)=\tau(x \xi e)=\lim _{i} \tau\left(y_{i} \xi e\right)=\lim _{i} \tau\left(e y_{i} \xi\right)=0
$$

which contradicts the fact that $\tau(e x \xi) \neq 0$. This ends the proof of part (i).

(ii) Suppose, to the contrary, that $[\mathcal{K} \cap \mathcal{M}]_{p} \varsubsetneqq \mathcal{K}$. Then there exists an $x \in \mathcal{K}$ such that $x \notin[\mathcal{K} \cap \mathcal{M}]_{p}$. Then, by the Hahn-Banach theorem, there exists $\varphi \in L^{p}(\mathcal{M}, \tau)^{\#}=L^{q}(\mathcal{M}, \tau)$ (where $\frac{1}{p}+\frac{1}{q}=1$ ), such that $\varphi(x) \neq 0$ and $\varphi(y)=0$ for every $y \in[\mathcal{K} \cap \mathcal{M}]_{p}$. This occurs if and only if there exists a $\xi \in L^{q}(\mathcal{M}, \tau)$ such that $\tau(x \xi) \neq 0$ and $\tau(y \xi)=0$ for every $y \in[\mathcal{K} \cap \mathcal{M}]_{p}$.

By Lemma 2.13, there exists a net $\left\{e_{\lambda}\right\}_{\lambda \in \Lambda}$ of projections in $\mathcal{D}$ such that $\tau\left(e_{\lambda}\right)<\infty$ for each $\lambda \in \Lambda$, and $\lim _{\lambda} \tau\left(e_{\lambda} x \xi\right)=\tau(x \xi)$. So, we may always assume that there exists a projection $e$ in $\mathcal{D}$ with $0<\tau(e)<\infty$ such that

(a) $\tau(e x \xi) \neq 0$; and

(b) $\tau($ ey $\xi)=0$ for every $y \in \mathcal{K} \cap \mathcal{M}$ (as $\mathcal{K}$ is $H^{\infty}$-invariant, and $e \in \mathcal{D} \subseteq H^{\infty}$ ).

Since $x \in L^{p}(\mathcal{M}, \tau)$ and $e$ is a projection in $\mathcal{D}$ such that $\tau(e)<\infty$, by Lemma 2.12, there exists a $h_{1} \in e H^{\infty} e$, and $h_{2} \in e H^{p} e$ such that $h_{1} e x \in \mathcal{M}$ and $h_{1} h_{2}=h_{2} h_{1}=e$. From the fact that $h_{2} \in e H^{p} e$, there exists a sequence $\left\{a_{n}\right\}_{n \in \mathbb{N}}$ in $e H^{\infty} e$ such that $\lim _{n \rightarrow \infty}\left\|a_{n}-h_{2}\right\|_{p}=0$. Therefore

$$
\begin{aligned}
\lim _{n \rightarrow \infty}\left|\tau\left(a_{n} h_{1} e x \xi\right)-\tau(e x \xi)\right| & =\lim _{n \rightarrow \infty}\left|\tau\left(a_{n} h_{1} e x \xi\right)-\tau\left(h_{2} h_{1} e x \xi\right)\right| \\
& \leq \lim _{n \rightarrow \infty}\left\|a_{n}-h_{2}\right\|\left\|_{p}\right\| h_{1} e x\|\| \xi \|_{q} \\
& =0 .
\end{aligned}
$$

On the other hand, since $a_{n}, h_{1}$ and $e$ are in $H^{\infty}$ and $h_{1} e x \in \mathcal{M}$, we know that $a_{n} h_{1} e x \in \mathcal{K} \cap \mathcal{M}$. From assumption (b), it follows that $\tau\left(a_{n} h_{1} e x \xi\right)=0$ for all $n \geq 1$. Therefore $\tau(e x \xi)=0$, which contradicts the assumption (a) that $\tau(x \xi e) \neq 0$. This ends the proof of part (ii). 
Proposition 4.2. Let $\mathcal{M}$ be a von Neumann algebra with a faithful, normal, semifinite tracial weight $\tau$ and $H^{\infty}$ be a semifinite subdiagonal subalgebra of $\mathcal{M}$.

Assume that $\mathcal{K} \subseteq \mathcal{M}$ is a weak $k^{*}$-closed subspace such that $H^{\infty} \mathcal{K} \subseteq \mathcal{K}$. Then

$$
\mathcal{K}=\overline{\left[\mathcal{K} \cap L^{p}(\mathcal{M}, \tau)\right]_{p} \cap \mathcal{M}^{w^{*}}}, \quad \forall 1 \leq p<\infty .
$$

ProOF. First, we show that

$$
\mathcal{K} \subseteq{\overline{\left[\mathcal{K} \cap L^{p}(\mathcal{M}, \tau)\right]_{p} \cap \mathcal{M}^{w *}}}^{w *}
$$

Let $x$ be an element in $\mathcal{K} \subseteq \mathcal{M}$. By Lemma 2.13, there exists a net $\left\{e_{\lambda}\right\}_{\lambda \in \Lambda}$ of projections in $\mathcal{D}$ such that such that $e_{\lambda} \rightarrow I$ in the weak* topology and $\tau\left(e_{\lambda}\right)<\infty$ for each $\lambda \in \Lambda$. By Lemma 2.1. $e_{\lambda} x \rightarrow x$ in the weak ${ }^{*}$ topology. To show that $x \in{\overline{\left[\mathcal{K} \cap L^{p}(\mathcal{M}, \tau)\right]_{p} \cap \mathcal{M}}}^{w *}$, it suffices to

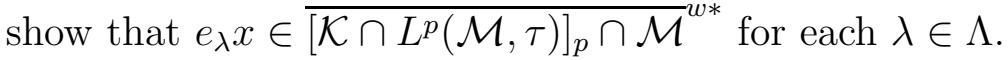

Since $\mathcal{K} \subseteq \mathcal{M}$ is left $H^{\infty}$-invariant and $x \in \mathcal{K}$, we have $e_{\lambda} x \in \mathcal{K}$. Moreover, $\left\|e_{\lambda} x\right\|_{p} \leq$ $\left\|e_{\lambda}\right\|{ }_{p}\|x\|<\infty$, so $e_{\lambda} x \in L^{p}(\mathcal{M}, \tau)$. It follows that $e_{\lambda} x \in \mathcal{K} \cap L^{p}(\mathcal{M}, \tau)$ for each $\lambda \in$

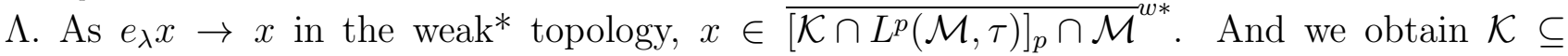

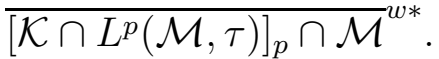

Next, we will show that

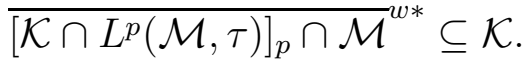

Since $\mathcal{K}$ is weak*-closed, it suffices to show that

$$
\left[\mathcal{K} \cap L^{p}(\mathcal{M}, \tau)\right]_{p} \cap \mathcal{M} \subseteq \mathcal{K}
$$

Assume, to the contrary, that $x$ is an element in $\left[\mathcal{K} \cap L^{p}(\mathcal{M}, \tau)\right]_{p} \cap \mathcal{M}$, but $x \notin \mathcal{K}$. Thus, by the Hahn-Banach theorem, there exists a weak* continuous linear functional $\varphi$ on $\mathcal{M}$ such that $\varphi(x) \neq 0$ and $\varphi(y)=0$ for every $y \in \mathcal{K}$. Or, there exists a $\xi \in L^{1}(\mathcal{M}, \tau)$ such that

(a) $\tau(x \xi) \neq 0$; and

(b) $\tau(y \xi)=0$ for every $y \in \mathcal{K}$.

By Lemma 2.13, there exists a net $\left\{e_{\lambda}\right\}_{\lambda \in \Lambda}$ of projections in $\mathcal{D}$ such that $\tau\left(e_{\lambda}\right)<\infty$ for each $\lambda \in \Lambda$ and $\lim _{\lambda} \tau\left(e_{\lambda} x \xi\right)=\tau(x \xi)$. So we may always assume that there exists a projection $e$ in $\mathcal{D}$ with $0<\tau(e)<\infty$ such that

$\left(\mathrm{a}_{1}\right) \tau(e x \xi) \neq 0$; and

$\left(\mathrm{b}_{1}\right) \tau(e y \xi)=0$ for every $y \in \mathcal{K}\left(\right.$ as $\mathcal{K}$ is $H^{\infty}$-invariant and $\left.e \in \mathcal{D} \subseteq H^{\infty}\right)$.

We claim there exists a $z=z e \in \mathcal{M} e$ such that

$\left(\mathrm{a}_{2}\right) \tau(x z) \neq 0$; and

$\left(\mathrm{b}_{2}\right) \tau(y z)=0$ for every $y \in \mathcal{K}$.

Observe that $\xi$ is in $L^{1}(\mathcal{M}, \tau)$, and $e$ is a projection in $\mathcal{D}$ such that $\tau(e)<\infty$. From Lemma 2.12, there exist $h_{3} \in e H^{\infty} e$ and $h_{4} \in e H^{1} e$ such that $\xi e h_{3} \in e \mathcal{M} e$ and $h_{3} h_{4}=e$. Thus there exists a sequence $\left\{k_{n}\right\}_{n \in \mathbb{N}}$ of elements in $e H^{\infty} e$ such that $\lim _{n \rightarrow \infty}\left\|k_{n}-h_{4}\right\|_{1}=0$. It follows that $\lim _{n \rightarrow \infty}\left|\tau(e x \xi)-\tau\left(x \xi e h_{3} k_{n}\right)\right|=\lim _{n \rightarrow \infty}\left|\tau\left(x \xi e h_{3} h_{4}\right)-\tau\left(x \xi e h_{3} k_{n}\right)\right| \leq \lim _{n \rightarrow \infty}\|x\|\left\|\xi e h_{3}\right\|\left\|h_{4}-k_{n}\right\|_{1}=0$. 
Combining this with $\left(\mathrm{a}_{1}\right)$, we know that there exists an $N \in \mathbb{N}$ such that $\tau\left(x \xi e h_{3} k_{N}\right) \neq 0$. Let $z=\left(\xi e h_{3}\right) k_{N}$ be in $\mathcal{M}$. Then $z=z e \in \mathcal{M} e$ satisfies

$\left(\mathrm{a}_{2}\right) \tau(x z)=\tau\left(x \xi e h_{3} k_{N}\right) \neq 0 ;$ and

$\left(\mathrm{b}_{2}\right) \tau(y z)=\tau\left(y \xi e h_{3} k_{N}\right)=\tau\left(\left(e h_{3} k_{N}\right) y \xi\right)=0$ for every $y \in \mathcal{K}$.

Note that $x \in\left[\mathcal{K} \cap L^{p}(\mathcal{M}, \tau)\right]_{p} \cap \mathcal{M}$. There exists a sequence $\left\{x_{n}\right\}_{n \in \mathcal{N}}$ in $\mathcal{K} \cap L^{p}(\mathcal{M}, \tau)$ such that $\lim _{n \rightarrow \infty}\left\|x_{n}-x\right\|_{p}=0$. Thus we have

$$
\left|\tau\left(x z-x_{n} z\right)\right|=\left|\tau\left(\left(x-x_{n}\right) z e\right)\right| \leq\left\|x_{n}-x\right\|_{p}\|z\|\|e\|_{q} \rightarrow 0,
$$

where $q$ satisfies $1 / p+1 / q=1$. On the other hand, since $\left\{x_{n}\right\}_{n \in \mathcal{N}}$ is in $\mathcal{K} \cap L^{p}(\mathcal{M}, \tau)$, by $\left(b_{2}\right)$ we have

$$
\tau\left(x_{n} z\right)=0, \quad \forall n \in \mathbb{N} .
$$

Combining with inequality (4.1), we have

$$
\tau(x z)=0 .
$$

This contradicts the assumption in $\left(\mathrm{a}_{2}\right)$ that $\tau(x z) \neq 0$. Therefore,

$$
{\overline{\left.\mathcal{K} \cap L^{p}(\mathcal{M}, \tau)\right]_{p} \cap \mathcal{M}^{w *} \subseteq \mathcal{K}}}^{w}
$$

Hence

$$
\mathcal{K}={\overline{\left[\mathcal{K} \cap L^{p}(\mathcal{M}, \tau)\right]_{p} \cap \mathcal{M}^{w *}}}^{w}
$$

LEMMA 4.3. If $u$ is a partial isometry in $\mathcal{M}$ such that $u u^{*} \in \mathcal{D}$, then

(i) $\left[\left(H^{\infty} u\right) \cap L^{p}(\mathcal{M}, \tau)\right]_{p}=H^{p} u$ for all $1 \leq p<\infty$, and

(ii) $H^{\infty} u={\overline{H^{p} u \cap \mathcal{M}}}^{w^{*}}$ for all $1 \leq p<\infty$.

Proof. (i) can be verified directly. (ii) follows from Proposition 4.2 and (i).

Proposition 4.4. Let $\mathcal{M}$ be a von Neumann algebra with a faithful, normal, semifinite tracial weight $\tau$ and let $H^{\infty}$ be a semifinite subdigonal subalgebra of $\mathcal{M}$. Assume that $S \subseteq \mathcal{M}$ is a subspace such that $H^{\infty} S \subseteq S$. Then

$$
\left[S \cap L^{p}(\mathcal{M}, \tau)\right]_{p}=\left[\bar{S}^{w^{*}} \cap L^{p}(\mathcal{M}, \tau)\right]_{p}, \quad \forall 1 \leq p<\infty .
$$

Proof. It suffices to show that

$$
\bar{S}^{w^{*}} \cap L^{p}(\mathcal{M}, \tau) \subseteq\left[S \cap L^{p}(\mathcal{M}, \tau)\right]_{p} .
$$

Let $x \in \bar{S}^{w^{*}} \cap L^{p}(\mathcal{M}, \tau)$. By Lemma 2.13, there exists a net $\left\{e_{\lambda}\right\}_{\lambda \in \Lambda}$ of projections in $\mathcal{D}$ such that $e_{\lambda} \rightarrow I$ in the weak* topology and $\tau\left(e_{\lambda}\right)<\infty$ for each $\lambda \in \Lambda$. By Lemma 2.6, $\lim _{\lambda}\left\|e_{\lambda} x-x\right\|_{p}=0$. To show that $x \in\left[S \cap L^{p}(\mathcal{M}, \tau)\right]_{p}$, it is enough to show that $e_{\lambda} x \in\left[S \cap L^{p}(\mathcal{M}, \tau)\right]_{p}$ for each $\lambda \in \Lambda$.

By Proposition 4.1, we have

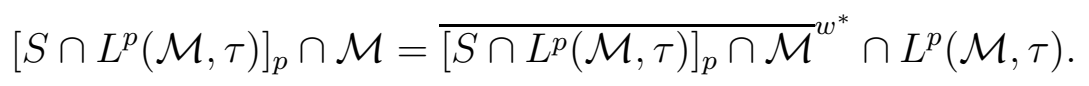


Since $x \in \bar{S}^{w^{*}} \cap L^{p}(\mathcal{M}, \tau)$, there exists a net $\left\{x_{j}\right\}_{j \in J}$ in $S$ such that $x_{j} \rightarrow x$ in weak ${ }^{*}$ topology. By Lemma 2.1, $e_{\lambda} x_{j} \rightarrow e_{\lambda} x$ in weak* topology for each $\lambda$. Note that $\left\|e_{\lambda} x_{j}\right\|_{p} \leq\left\|e_{\lambda}\right\|\left\|_{p}\right\| x_{j} \|$ and $H^{\infty} S \subseteq S$. We know that $e_{\lambda} x_{j} \in S \cap L^{p}(\mathcal{M}, \tau)$. So $e_{\lambda} x$ is in ${\overline{\left[S \cap L^{p}(\mathcal{M}, \tau)\right]_{p} \cap \mathcal{M}}}^{w^{*}}$. It is trivial to see that $e_{\lambda} x \in L^{p}(\mathcal{M}, \tau)$. Hence,

$$
e_{\lambda} x \in \overline{\left[S \cap L^{p}(\mathcal{M}, \tau)\right]_{p} \cap \mathcal{M}^{w^{*}}} \cap L^{p}(\mathcal{M}, \tau)=\left[S \cap L^{p}(\mathcal{M}, \tau)\right]_{p} .
$$

So

$$
x \in\left[S \cap L^{p}(\mathcal{M}, \tau)\right]_{p}
$$

Thus

$$
\bar{S}^{w^{*}} \cap L^{p}(\mathcal{M}, \tau) \subseteq\left[S \cap L^{p}(\mathcal{M}, \tau)\right]_{p}
$$

Hence

$$
\left[S \cap L^{p}(\mathcal{M}, \tau)\right]_{p}=\left[\bar{S}^{w^{*}} \cap L^{p}(\mathcal{M}, \tau)\right]_{p}, \quad \forall 1 \leq p<\infty
$$

THEOREM 4.5. Let $\mathcal{M}$ be a von Neumann algebra with a faithful, normal, semifinite tracial weight $\tau$, and $H^{\infty}$ be a semifinite subdigonal subalgebra of $\mathcal{M}$. Let $\mathcal{D}=H^{\infty} \cap\left(H^{\infty}\right)^{*}$. Assume that $\mathcal{K} \subseteq \mathcal{M}$ is weak $k^{*}$-closed subspace such that $H^{\infty} \mathcal{K} \subseteq \mathcal{K}$.

Then there exist a weak ${ }^{*}$ closed subspace $Y$ of $\mathcal{M}$ and a family $\left\{u_{\lambda}\right\}_{\lambda \in \Lambda}$ of partial isometries in $\mathcal{M}$ such that:

(i) $u_{\lambda} Y^{*}=0$ for all $\lambda \in \Lambda$.

(ii) $u_{\lambda} u_{\lambda}^{*} \in \mathcal{D}$ and $u_{\lambda} u_{\mu}^{*}=0$ for all $\lambda, \mu \in \Lambda$ with $\lambda \neq \mu$.

(iii) $Y={\overline{H_{0}^{\infty}}}^{w^{*}}$.

(iv) $\mathcal{K}=Y \oplus^{\text {row }}\left(\oplus_{\lambda \in \Lambda}^{\text {row }} H^{\infty} u_{\lambda}\right)$

Here $\oplus^{\text {row }}$ is the row sum of subspaces defined in Definition 2.15.

Proof. Let $\mathcal{K}_{1}=\left[\mathcal{K} \cap L^{2}(\mathcal{M}, \tau)\right]_{2}$. Then $\mathcal{K}_{1}$ is a closed subspace of $L^{2}(\mathcal{M}, \tau)$ such that $H^{\infty} \mathcal{K}_{1} \subseteq \mathcal{K}_{1}$. By Theorem 3.1, there exist a closed subspace $Y_{1}$ of $L^{2}(\mathcal{M}, \tau)$ and a family $\left\{u_{\lambda}\right\}_{\lambda \in \Lambda}$ of partial isometries in $\mathcal{M}$, satisfying

(a) $u_{\lambda} Y_{1}^{*}=0$ for all $\lambda \in \Lambda$.

(b) $u_{\lambda} u_{\lambda}^{*} \in \mathcal{D}$ and $u_{\lambda} u_{\mu}^{*}=0$ for all $\lambda, \mu \in \Lambda$ with $\lambda \neq \mu$.

(c) $Y_{1}=\left[H_{0}^{\infty} Y_{1}\right]_{2}$, where $H_{0}^{\infty}=H^{\infty} \cap \operatorname{ker}(\Phi)$.

(d) $\mathcal{K}_{1}=Y_{1} \oplus\left(\oplus_{\lambda \in \Lambda} H^{2} u_{\lambda}\right)$

Let

$$
Y=\bar{Y}_{1 \cap \mathcal{M}^{w^{*}}}
$$

(i) We show that (i) is satisfied. In fact, from (a) and Lemma 2.1, we have

$$
u_{\lambda} Y^{*}=0 \text { for all } \lambda \in \Lambda \text {. }
$$

(ii) follows directly from (b), i.e.

$$
u_{\lambda} u_{\lambda}^{*} \in \mathcal{D} \text { and } u_{\lambda} u_{\mu}^{*}=0 \text { for all } \lambda, \mu \in \Lambda \text { with } \lambda \neq \mu .
$$


(iii) We claim that

$$
Y={\overline{H_{0}^{\infty}}}^{w^{*}} .
$$

In fact, we need only to show that $Y \subseteq{\overline{H_{0}^{\infty} Y}}^{w^{*}}$. By Proposition 4.1 and the definition of $Y$, we have

$$
Y_{1}=\left[Y_{1} \cap \mathcal{M}\right]_{2}=\left[\bar{Y}, \cap \mathcal{M}^{w *} \cap L^{2}(\mathcal{M}, \tau)\right]_{2}=\left[Y \cap L^{2}(\mathcal{M}, \tau)\right]_{2}
$$

So

$$
H_{0}^{\infty} Y_{1}=H_{0}^{\infty}\left[Y \cap L^{2}(\mathcal{M}, \tau)\right]_{2} \subseteq\left[\left(H_{0}^{\infty} Y\right) \cap L^{2}(\mathcal{M}, \tau)\right]_{2} \subseteq\left[{\overline{H_{0}^{\infty}}}^{w^{*}} \cap L^{2}(\mathcal{M}, \tau)\right]_{2} .
$$

Thus, from (c), we have

$$
Y_{1}=\left[H_{0}^{\infty} Y_{1}\right]_{2} \subseteq\left[{\overline{H_{0}^{\infty}}}^{w^{*}} \cap L^{2}(\mathcal{M}, \tau)\right]_{2} .
$$

Now, we are able to conclude that

$$
\begin{aligned}
Y & ={\overline{Y_{1} \cap \mathcal{M}}}^{w^{*}} \\
& \subseteq \overline{\left[\bar{H}_{0}^{\infty} Y^{w^{*}} \cap L^{2}(\mathcal{M}, \tau)\right]_{2} \cap \mathcal{M}} w^{*} \\
& ={\overline{H_{0}^{\infty} Y}}^{w^{*}} .
\end{aligned}
$$

(by Proposition 4.2)

Thus

$$
Y={\overline{H_{0}^{\infty} Y}}^{w^{*}}
$$

(iv) We show that

$$
\overline{\operatorname{span}\left\{Y_{2}, H^{\infty} u_{\lambda}: \lambda \in \Lambda\right\}}{ }^{w^{*}}=\mathcal{K} .
$$

By Proposition 4.2, it suffices to show that

$$
\overline{\operatorname{span}\left\{Y, H^{\infty} u_{\lambda}: \lambda \in \Lambda\right\}}{ }^{w^{*}}={\overline{\left[\mathcal{K} \cap L^{2}(\mathcal{M}, \tau)\right]_{2} \cap \mathcal{M}}}^{w^{*}}=\mathcal{K} .
$$

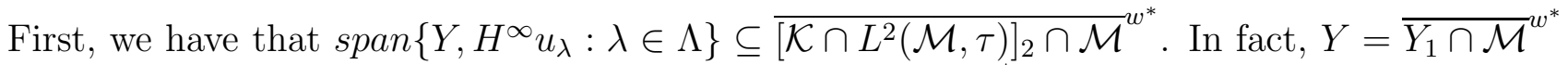
and $Y_{1} \subseteq\left[\mathcal{K} \cap L^{2}(\mathcal{M}, \tau)\right]_{2}$, so $Y \subseteq{\overline{\left[\mathcal{K} \cap L^{2}(\mathcal{M}, \tau)\right]_{2} \cap \mathcal{M}}}^{w^{*}}$. Moreover, for each $\lambda \in \Lambda$, by Lemma 4.3, we have $H^{\infty} u_{\lambda}={\overline{H^{2} u_{\lambda} \cap \mathcal{M}}}^{w^{*}} \subseteq{\overline{\left[\mathcal{K} \cap L^{2}(\mathcal{M}, \tau)\right]_{2} \cap \mathcal{M}}}^{w^{*}}$. So

$$
\operatorname{span}\left\{Y, H^{\infty} u_{\lambda}: \lambda \in \Lambda\right\} \subseteq{\overline{\left[\mathcal{K} \cap L^{2}(\mathcal{M}, \tau)\right]_{2} \cap \mathcal{M}^{w *}}}^{w^{*}}
$$

Thus

$$
\overline{\operatorname{span}\left\{Y, H^{\infty} u_{\lambda}: \lambda \in \Lambda\right\}} w^{*} \subseteq{\overline{\left[\mathcal{K} \cap L^{2}(\mathcal{M}, \tau)\right]_{2} \cap \mathcal{M}}}^{w^{*}}=\mathcal{K}
$$

Next, define $X=\overline{\operatorname{span}\left\{Y, H^{\infty} u_{\lambda}: \lambda \in \Lambda\right\}}{ }^{w^{*}}$. We want to show that

$$
\overline{\left[\mathcal{K} \cap L^{2}(\mathcal{M}, \tau)\right]_{2} \cap \mathcal{M}^{w^{*}} \subseteq X .}
$$


Notice $X$ is weak*-closed and $H^{\infty} X \subseteq X$. By Proposition 4.2, $X=\overline{\left[X \cap L^{2}(\mathcal{M}, \tau)\right]_{2} \cap \mathcal{M}} w^{*}$. Therefore we need only to show that $\left[\mathcal{K} \cap L^{2}(\mathcal{M}, \tau)\right]_{2} \subseteq\left[X \cap L^{2}(\mathcal{M}, \tau)\right]_{2}$. Or, equivalently, we may show $Y_{1}$ and $\left\{H^{2} u_{\lambda}\right\}_{\lambda \in \Lambda}$ are in $\left[X \cap L^{2}(\mathcal{M}, \tau)\right]_{2}$. By Proposition 4.1, we have

$$
Y_{1}=\left[Y_{1} \cap \mathcal{M}\right]_{2}=\left[\bar{Y}_{1} \cap \mathcal{M}^{w *} \cap L^{2}(\mathcal{M}, \tau)\right]_{2}=\left[Y \cap L^{2}(\mathcal{M}, \tau)\right]_{2} .
$$

Thus

$$
Y_{1} \subseteq\left[X \cap L^{2}(\mathcal{M}, \tau)\right]_{2}
$$

By Lemma 4.3 ,

$$
H^{2} u_{\lambda}=\left[H^{\infty} u_{\lambda} \cap L^{2}(\mathcal{M}, \tau)\right]_{2} \subseteq\left[X \cap L^{2}(\mathcal{M}, \tau)\right]_{2} \quad \text { for each } \lambda \in \Lambda .
$$

Hence, from (4.7) and (4.8), we get $\left[\mathcal{K} \cap L^{2}(\mathcal{M}, \tau)\right]_{2} \subseteq\left[X \cap L^{2}(\mathcal{M}, \tau)\right]_{2}$ and

$$
\mathcal{K}={\overline{\left[\mathcal{K} \cap L^{2}(\mathcal{M}, \tau)\right]_{2} \cap \mathcal{M}}}^{w^{*}} \subseteq \overline{\operatorname{span}\left\{Y, H^{\infty} u_{\lambda} \lambda \in \Lambda\right\}}{ }^{w^{*}} .
$$

Now, combining (4.6) and (4.9), we have

$$
\mathcal{K}=\overline{\operatorname{span}\left\{Y, H^{\infty} u_{\lambda} \lambda \in \Lambda\right\}} w^{w^{*}}=Y \oplus^{\text {row }}\left(\oplus_{\lambda \in \Lambda}^{\text {row }} H^{\infty} u_{\lambda}\right),
$$

by the definition of row sum of subspaces.

By (4.10), (4.2), (4.3) and (4.5), we know that $Y$ and $\left\{u_{\lambda}\right\}_{\lambda \in \Lambda}$ have the desired properties. This ends the proof of the theorem.

Next, we use our result for $p=\infty$ and the density theorem to prove the case when $1 \leq p<\infty$.

TheOrem 4.6. Let $1 \leq p<\infty$. Let $\mathcal{M}$ be a von Neumann algebra with a faithful, normal, semifinite tracial weight $\tau$, and $H^{\infty}$ be a semifinite subdigonal subalgebra of $\mathcal{M}$. Let $\mathcal{D}=$ $H^{\infty} \cap\left(H^{\infty}\right)^{*}$. Assume that $\mathcal{K}$ is a closed subspace of $L^{p}(\mathcal{M}, \tau)$ such that $H^{\infty} \mathcal{K} \subseteq \mathcal{K}$.

Then there exist a closed subspace $Y$ of $L^{p}(\mathcal{M}, \tau)$ and a family $\left\{u_{\lambda}\right\}_{\lambda \in \Lambda}$ of partial isometries in $\mathcal{M}$ such that:

(i) $u_{\lambda} Y^{*}=0$ for all $\lambda \in \Lambda$.

(ii) $u_{\lambda} u_{\lambda}^{*} \in \mathcal{D}$ and $u_{\lambda} u_{\mu}^{*}=0$ for all $\lambda, \mu \in \Lambda$ with $\lambda \neq \mu$.

(iii) $Y=\left[H_{0}^{\infty} Y\right]_{p}$.

(iv) $\mathcal{K}=Y \oplus^{\text {row }}\left(\oplus_{\lambda \in \Lambda}^{\text {row }} H^{p} u_{\lambda}\right)$

Here $\oplus^{\text {row }}$ is the row sum of subspaces defined in Definition 2.15.

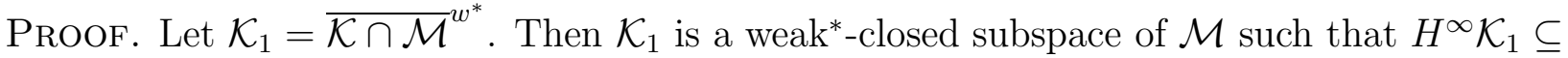
$\mathcal{K}_{1}$. By Theorem 4.5, there exist a weak*-closed subspace $Y_{1}$ of $\mathcal{M}$ and a family $\left\{u_{\lambda}\right\}_{\lambda \in \Lambda}$ of partial isometries in $\mathcal{M}$, satisfying

(a) $u_{\lambda} Y_{1}^{*}=0$ for all $\lambda \in \Lambda$.

(b) $u_{\lambda} u_{\lambda}^{*} \in \mathcal{D}$ and $u_{\lambda} u_{\mu}^{*}=0$ for all $\lambda, \mu \in \Lambda$ with $\lambda \neq \mu$.

(c) $Y_{1}={\overline{H_{0}^{\infty} Y_{1}}}^{w^{*}}$.

(d) $\mathcal{K}_{1}=Y_{1} \oplus^{\text {row }}\left(\oplus_{\lambda \in \Lambda}^{\text {row }} H^{\infty} u_{\lambda}\right)$ 
Let

$$
Y=\left[Y_{1} \cap L^{p}(\mathcal{M}, \tau)\right]_{p}
$$

(i) From (a), the definition of $Y$ and Lemma 2.5, we can conclude that

$$
u_{\lambda} Y^{*}=0 \text { for all } \lambda \in \Lambda \text {. }
$$

(ii) follows directly from (b), i.e.

$$
u_{\lambda} u_{\lambda}^{*} \in \mathcal{D} \text { and } u_{\lambda} u_{\mu}^{*}=0 \text { for all } \lambda, \mu \in \Lambda \text { with } \lambda \neq \mu \text {. }
$$

(iii) We want to show that $Y=\left[H_{0}^{\infty} Y\right]_{p}$. In fact, we have

$$
\begin{aligned}
& Y=\left[Y_{1} \cap L^{p}(\mathcal{M}, \tau)\right]_{p} \\
& =\left[{\overline{H_{0}^{\infty} Y_{1}}}^{w^{*}} \cap L^{p}(\mathcal{M}, \tau)\right]_{p} \\
& =\left[\left(H_{0}^{\infty} Y_{1}\right) \cap L^{p}(\mathcal{M}, \tau)\right]_{p} \\
& =\left[\left(H_{0}^{\infty} \overline{\left[Y_{1} \cap L^{p}(\mathcal{M}, \tau)\right]_{p} \cap \mathcal{M}^{w^{*}}}\right) \cap L^{p}(\mathcal{M}, \tau)\right]_{p} \\
& \subseteq\left[{\overline{H_{0}^{\infty}}\left(\left[Y_{1} \cap L^{p}(\mathcal{M}, \tau)\right]_{p} \cap \mathcal{M}\right)}^{w^{*}} \cap L^{p}(\mathcal{M}, \tau)\right]_{p} \\
& =\left[\left(H_{0}^{\infty}\left(\left[Y_{1} \cap L^{p}(\mathcal{M}, \tau)\right]_{p} \cap \mathcal{M}\right)\right) \cap L^{p}(\mathcal{M}, \tau)\right]_{p} \\
& =\left[\left(H_{0}^{\infty}(Y \cap \mathcal{M})\right) \cap L^{p}(\mathcal{M}, \tau)\right]_{p} \\
& \subseteq\left[H_{0}^{\infty} Y\right]_{p} \subseteq Y,
\end{aligned}
$$

(by Lemma 2.1) (by Proposition 4.4)

(iv) There is only left to show that

$$
\mathcal{K}=Y \oplus^{\text {row }}\left(\oplus_{\lambda \in \Lambda}^{\text {row }} H^{p} u_{\lambda}\right) .
$$

By the definition of $Y$, we have

$$
Y=\left[Y_{1} \cap L^{p}(\mathcal{M}, \tau)\right]_{p}
$$

And from Lemma 4.3, we have

$$
H^{p} u_{\lambda}=\left[H^{\infty} u_{\lambda} \cap L^{p}(\mathcal{M}, \tau)\right]_{p}, \quad \forall \lambda \in \Lambda .
$$

Now, we have

$$
\begin{array}{rlr}
\mathcal{K} & =\left[\mathcal{K}_{1} \cap L^{p}(\mathcal{M}, \tau)\right]_{p} & \text { (by Proposition 4.1) } \\
& =\left[\overline{\operatorname{span}\left\{Y_{1}, H^{\infty} u_{\lambda}: \lambda \in \Lambda\right\}}{ }^{w^{*}} \cap L^{p}(\mathcal{M}, \tau)\right]_{p} & \text { (by the definition of row sum of subspaces) } \\
& =\left[\operatorname{span}\left\{Y_{1}, H^{\infty} u_{\lambda}: \lambda \in \Lambda\right\} \cap L^{p}(\mathcal{M}, \tau)\right]_{p} & \text { (by Proposition 4.4) } \\
& =\left[\operatorname{span}\left\{Y_{1} \cap L^{p}(\mathcal{M}, \tau), H^{\infty} u_{\lambda} \cap L^{p}(\mathcal{M}, \tau): \lambda \in \Lambda\right\}\right]_{p} & \text { (by (a) and (b)) } \\
& =\left[\operatorname{span}\left\{Y, H^{p} u_{\lambda}: \lambda \in \Lambda\right\}\right]_{p} & \text { (by (4.14) and (4.15)) } \\
& =Y \oplus^{\text {row }}\left(\oplus_{\lambda \in \Lambda}^{\text {row }} H^{p} u_{\lambda}\right),
\end{array}
$$

where the last equation follows from the definition of the row sum of subspaces.

As a summary, from (4.11), (4.12), (4.13), and (4.16), $Y$ and $\left\{u_{\lambda}\right\}_{\lambda \in \Lambda}$ have the desired properties. This ends the proof of the theorem. 
5. Beurling-Blecher-Labuschagne Theorem for Semifinite Hardy Spaces, $0<p<1$

\subsection{Dense subspaces.}

Proposition 5.1. Suppose $0<p<1$. Let $\mathcal{M}$ be a von Neumann algebra with a faithful, normal, semifinite tracial weight $\tau$, and $H^{\infty}$ be a semifinite subdigonal subalgebra of $\mathcal{M}$. Assume that $\mathcal{K}$ is a closed subspace in $L^{p}(\mathcal{M}, \tau)$ such that $H^{\infty} \mathcal{K} \subseteq \mathcal{K}$. Then the following statements are true.

(i) $\mathcal{K} \cap L^{2}(\mathcal{M}, \tau)=\left[\mathcal{K} \cap L^{2}(\mathcal{M}, \tau)\right]_{2} \cap L^{p}(\mathcal{M}, \tau)$.

(ii) $\mathcal{K}=\left[\mathcal{K} \cap L^{2}(\mathcal{M}, \tau)\right]_{p}$.

Proof. (i) We need only to show that

$$
\left[\mathcal{K} \cap L^{2}(\mathcal{M}, \tau)\right]_{2} \cap L^{p}(\mathcal{M}, \tau) \subseteq \mathcal{K} \cap L^{2}(\mathcal{M}, \tau) .
$$

Let $x \in\left[K_{1} \cap L^{2}(\mathcal{M}, \tau)\right]_{2} \cap L^{p}(\mathcal{M}, \tau)$. We will show that $x \in \mathcal{K}$. By Lemma 2.13, there exists a net $\left\{e_{\lambda}\right\}_{\lambda \in \Lambda}$ of projections in $\mathcal{D}$ such that such that $\tau\left(e_{\lambda}\right)<\infty$ for each $\lambda \in \Lambda$ and $\lim _{\lambda}\left\|e_{\lambda} x-x\right\|_{p}=0$. To show that $x \in \mathcal{K}$, it is enough to prove that $e_{\lambda} x \in \mathcal{K}$ for each $\lambda \in \Lambda$.

As $x \in\left[\mathcal{K} \cap L^{2}(\mathcal{M}, \tau)\right]_{2}$, there exists a sequence $\left\{x_{n}\right\}_{n \in \mathbb{N}}$ in $K_{1} \cap L^{2}(\mathcal{M}, \tau)$ such that $\lim _{n \rightarrow \infty}\left\|x_{n} \rightarrow x\right\|_{2}=0$. Thus, for each $\lambda \in \Lambda$ and some positive number $q$ with $\frac{1}{2}+\frac{1}{q}=\frac{1}{p}$,

$$
\lim _{n \rightarrow \infty}\left\|e_{\lambda} x_{n}-e_{\lambda} x\right\|_{p}=\lim _{n \rightarrow \infty}\left\|e_{\lambda}\left(x_{n}-x\right)\right\|_{p} \leq \lim _{n \rightarrow \infty}\left\|x_{n}-x\right\|_{2}\left\|e_{\lambda}\right\|_{q}=0 .
$$

Here, we used the fact that $\tau\left(e_{\lambda}\right)<\infty$ and $\left\|e_{\lambda}\right\|_{q}<\infty$. Since $H^{\infty} \mathcal{K} \subseteq \mathcal{K}$ and $e_{\lambda} \in \mathcal{D}$, we know that $e_{\lambda} x_{n} \in \mathcal{K}$. This implies that $e_{\lambda} x \in \mathcal{K}$ for each $\lambda \in \Lambda$. Thus $x \in \mathcal{K}$, whence

$$
\left[\mathcal{K} \cap L^{2}(\mathcal{M}, \tau)\right]_{2} \cap L^{p}(\mathcal{M}, \tau) \subseteq \mathcal{K} \cap L^{2}(\mathcal{M}, \tau)
$$

(ii) We need only to show that

$$
\mathcal{K} \subseteq\left[\mathcal{K} \cap L^{2}(\mathcal{M}, \tau)\right]_{p}
$$

Suppose that $x \in \mathcal{K} \subseteq L^{p}(\mathcal{M}, \tau)$. We will show $x \in\left[\mathcal{K} \cap L^{2}(\mathcal{M}, \tau)\right]_{p}$. By Lemma 2.13, we can find a net $\left\{e_{\lambda}\right\}_{\lambda \in \Lambda}$ of projections in $\mathcal{D}$ such that $\lim _{\lambda}\left\|e_{\lambda} x-x\right\|_{2}=0$ and $\tau\left(e_{\lambda}\right)<\infty$ for each $\lambda \in \Lambda$. To show that $x \in\left[\mathcal{K} \cap L^{2}(\mathcal{M}, \tau)\right]_{p}$, it suffices to prove that $e_{\lambda} x \in\left[\mathcal{K} \cap L^{2}(\mathcal{M}, \tau)\right]_{p}$ for each $\lambda \in \Lambda$.

Note that $x \in L^{p}(\mathcal{M}, \tau)$ and $\tau\left(e_{\lambda}\right)<\infty$. By Lemma 2.12, there exist $h_{1} \in e_{\lambda} H^{\infty} e_{\lambda}$ and $h_{2} \in e_{\lambda} H^{p} e_{\lambda}$ such that (a) $h_{1} h_{2}=h_{2} h_{1}=e_{\lambda}$ and (b) $h_{1} e_{\lambda} x \in \mathcal{M}$. Since $h_{2} \in e_{\lambda} H^{p} e_{\lambda}$, there exists a sequence $\left\{k_{n}\right\}_{n \in \mathbb{N}}$ in $e_{\lambda} H^{\infty} e_{\lambda}$ such that $\lim _{n \rightarrow \infty}\left\|k_{n}-h_{2}\right\|_{p}=0$. Thus

$$
\lim _{n \rightarrow \infty}\left\|k_{n} h_{1} e_{\lambda} x-e_{\lambda} x\right\|_{p}=\lim _{n \rightarrow \infty}\left\|\left(k_{n}-h_{2}\right) h_{1} e_{\lambda} x\right\|_{p} \leq \lim _{n \rightarrow \infty}\left\|\left(k_{n}-h_{2}\right)\right\|_{p}\left\|h_{1} e_{\lambda} x\right\|=0 .
$$

It is not hard to check that $k_{n} h_{1} e_{\lambda} x \in \mathcal{K}$. Moreover, since each $k_{n} \in e_{\lambda} H^{\infty} e_{\lambda}$, we have

$$
\left\|k_{n} h_{1} e_{\lambda} x\right\|_{p}=\left\|e_{\lambda} k_{n} h_{1} e_{\lambda} x\right\|_{2} \leq\left\|e_{\lambda}\right\|_{2}\left\|k_{n}\right\|\left\|h_{1} e_{\lambda} x\right\|<\infty .
$$

Therefore, $k_{n} h_{1} e_{\lambda} x$ is also in $L^{2}(\mathcal{M}, \tau)$. It follows that $k_{n} h_{1} e_{\lambda} x \in \mathcal{K} \cap L^{p}(\mathcal{M}, \tau)$. Combining with (5.1), we know that $e_{\lambda} x \in\left[\mathcal{K} \cap L^{2}(\mathcal{M}, \tau)\right]_{p}$ for each $\lambda \in \Lambda$, whence $x \in\left[\mathcal{K} \cap L^{2}(\mathcal{M}, \tau)\right]_{p}$. Thus

$$
\mathcal{K} \subseteq\left[\mathcal{K} \cap L^{2}(\mathcal{M}, \tau)\right]_{p}
$$


This ends the proof of the proposition.

Proposition 5.2. Suppose $0<p<1$. Let $\mathcal{M}$ be a von Neumann algebra with a faithful, normal, semifinite tracial weight $\tau$, and $H^{\infty}$ be a semifinite subdigonal subalgebra of $\mathcal{M}$. Assume that $S$ is a subspace in $L^{p}(\mathcal{M}, \tau)$ such that $H^{\infty} S \subseteq S$. Then

$$
\left[S \cap L^{p}(\mathcal{M}, \tau)\right]_{p}=\left[[S]_{2} \cap L^{p}(\mathcal{M}, \tau)\right]_{p} .
$$

Proof. We need only to show that

$$
\left[[S]_{2} \cap L^{p}(\mathcal{M}, \tau)\right]_{p} \subseteq\left[S \cap L^{p}(\mathcal{M}, \tau)\right]_{p} .
$$

Or, equivalently,

$$
[S]_{2} \cap L^{p}(\mathcal{M}, \tau) \subseteq\left[S \cap L^{p}(\mathcal{M}, \tau)\right]_{p}
$$

Let $x \in[S]_{2} \cap L^{p}(\mathcal{M}, \tau)$. By Lemma 2.13 , we can find a net $\left\{e_{\lambda}\right\}_{\lambda \in \Lambda}$ of projections in $\mathcal{D}$ such that $\lim _{\lambda}\left\|e_{\lambda} x-x\right\|_{p}=0$ and $\tau\left(e_{\lambda}\right)<\infty$ for each $\lambda \in \Lambda$. To show that $x \in\left[S \cap L^{p}(\mathcal{M}, \tau)\right]_{p}$, it suffices to prove that $e_{\lambda} x \in\left[S \cap L^{p}(\mathcal{M}, \tau)\right]_{p}$ for each $\lambda \in \Lambda$.

Note that $x \in[S]_{2} \cap L^{p}(\mathcal{M}, \tau)$. Then there exists a sequence $\left\{x_{n}\right\}_{n \in \mathbb{N}}$ in $S$ such that $\lim _{n \rightarrow \infty}\left\|x_{n}-x\right\|_{2}=0$. Therefore,

$$
\left\|e_{\lambda} x_{n}-e_{\lambda} x\right\|_{p}=\left\|e_{\lambda}\left(x_{n}-x\right)\right\|_{p} \leq\left\|e_{\lambda}\right\|_{q}\left\|x_{n}-x\right\|_{2} \rightarrow 0, \quad \text { as } n \rightarrow \infty,
$$

where $q$ is a positive number such that $\frac{1}{2}+\frac{1}{q}=\frac{1}{p}$. Since $H^{\infty} S \subseteq S$ and $e_{\lambda} \in \mathcal{D}$, we know that $e_{\lambda} x_{n} \in S$. Moreover, $\left\|e_{\lambda} x_{n}\right\|_{p} \leq\left\|e_{\lambda}\right\|_{q}\left\|x_{n}\right\|_{2}<\infty$, which implies $e_{\lambda} x_{n} \in L^{p}(\mathcal{M}, \tau)$. This induces that $e_{\lambda} x_{n} \in S \cap L^{p}(\mathcal{M}, \tau)$. Combining with (15.2), we have that $e_{\lambda} x \in\left[S \cap L^{p}(\mathcal{M}, \tau)\right]_{p}$ for each $\lambda \in \Lambda$. Thus $x \in\left[S \cap L^{p}(\mathcal{M}, \tau)\right]_{p}$ for each $\lambda \in \Lambda$. Or,

$$
[S]_{2} \cap L^{p}(\mathcal{M}, \tau) \subseteq\left[S \cap L^{p}(\mathcal{M}, \tau)\right]_{p}
$$

This ends the proof of the proposition.

LEMMA 5.3. If $u$ is a partial isometry in $\mathcal{M}$ such that $u u^{*} \in \mathcal{D}$, then

(i) $\left[\left(H^{2} u\right) \cap L^{p}(\mathcal{M}, \tau)\right]_{p}=H^{p} u \quad$ for $0<p<1$;

(ii) $H^{2} u=\left[H^{p} u \cap L^{2}(\mathcal{M}, \tau)\right]_{2} \quad$ for $0<p<1$.

Proof. (i) Assume that $x \in H^{2}$ such that $x u \in\left(H^{2} u\right) \cap L^{p}(\mathcal{M}, \tau)$. Then $x u u^{*} \in L^{p}(\mathcal{M}, \tau)$, and $x\left(u u^{*}\right)$ is also in $H^{2}$, as $u u^{*} \in \mathcal{D}$. So $x u u^{*} \in H^{2} \cap L^{p}(\mathcal{M}, \tau) \subseteq H^{p}$ by Proposition 3.2 in [5]. Note that $H^{p} u$ is a closed subspace in $L^{p}(\mathcal{M}, \tau)$. We have

$$
\left[\left(H^{2} u\right) \cap L^{p}(\mathcal{M}, \tau)\right]_{p} \subseteq H^{p} u
$$

Similarly, we have

$$
\left[\left(H^{p} u\right) \cap L^{2}(\mathcal{M}, \tau)\right]_{2} \subseteq H^{2} u
$$

Combining with Proposition 5.1, we have

$$
H^{p} u=\left[H^{p} u \cap L^{2}(\mathcal{M}, \tau)\right]_{p} \subseteq\left[\left(H^{2} u\right) \cap L^{p}(\mathcal{M}, \tau)\right]_{p} .
$$

Hence $\left[\left(H^{2} u\right) \cap L^{p}(\mathcal{M}, \tau)\right]_{p}=H^{p} u$, for $0<p<1$. 
(ii) Let $x \in H^{2}$. By Lemma 2.13, we can find a net $\left\{e_{\lambda}\right\}_{\lambda \in \Lambda}$ of projections in $\mathcal{D}$ such that $\lim _{\lambda}\left\|e_{\lambda} x-x\right\|_{2}=0$ and $\tau\left(e_{\lambda}\right)<\infty$ for each $\lambda \in \Lambda$. From $\tau\left(e_{\lambda}\right)<\infty$, it is easy to verify that $e_{\lambda} x \in L^{p}(\mathcal{M}, \tau) \cap H^{2} \subseteq H^{p}$ by Proposition 3.2 in 5. Thus $e_{\lambda} x u \in\left(H^{p} u\right) \cap L^{2}(\mathcal{M}, \tau)$ for each $\lambda \in \Lambda$, whence $x u \in\left[\left(H^{p} u\right) \cap L^{2}(\mathcal{M}, \tau)\right]_{2}$. Or,

$$
H^{2} u \subseteq\left[\left(H^{p} u\right) \cap L^{2}(\mathcal{M}, \tau)\right]_{2} .
$$

Combining with what we proved in (i), we have

$$
H^{2} u=\left[\left(H^{p} u\right) \cap L^{2}(\mathcal{M}, \tau)\right]_{2} .
$$

Now, we can prove a Beurling-Blecher-Labuschagne Theorem for the semifinite case when $0<p<1$.

TheORem 5.4. Let $0<p<1$. Let $\mathcal{M}$ be a von Neumann algebra with a faithful, normal, semifinite tracial weight $\tau$, and $H^{\infty}$ be a semifinite subdigonal subalgebra of $\mathcal{M}$. Let $\mathcal{D}=$ $H^{\infty} \cap\left(H^{\infty}\right)^{*}$. Assume that $\mathcal{K}$ is a closed subspace of $L^{p}(\mathcal{M}, \tau)$ such that $H^{\infty} \mathcal{K} \subseteq \mathcal{K}$.

Then there exist a closed subspace $Y$ of $L^{p}(\mathcal{M}, \tau)$ and a family $\left\{u_{\lambda}\right\}_{\lambda \in \Lambda}$ of partial isometries in $\mathcal{M}$ such that:

(i) $u_{\lambda} Y^{*}=0$ for all $\lambda \in \Lambda$.

(ii) $u_{\lambda} u_{\lambda}^{*} \in \mathcal{D}$ and $u_{\lambda} u_{\mu}^{*}=0$ for all $\lambda, \mu \in \Lambda$ with $\lambda \neq \mu$.

(iii) $Y=\left[H_{0}^{\infty} Y\right]_{p}$.

(iv) $\mathcal{K}=Y \oplus^{\text {row }}\left(\oplus_{\lambda \in \Lambda}^{\text {row }} H^{p} u_{\lambda}\right)$

Here $\oplus^{\text {row }}$ is the row sum of subspaces defined in Definition 2.14.

Proof. Let $\mathcal{K}_{1}=\left[\mathcal{K} \cap L^{2}(\mathcal{M}, \tau)\right]_{2}$. Then $\mathcal{K}_{1}$ is a closed subspace of $L^{2}(\mathcal{M}, \tau)$ such that $H^{\infty} \mathcal{K}_{1} \subseteq \mathcal{K}_{1}$. By Theorem 4.6, there exist a closed subspace $Y_{1}$ of $L^{2}(\mathcal{M}, \tau)$ and a family $\left\{u_{\lambda}\right\}_{\lambda \in \Lambda}$ of partial isometries in $\mathcal{M}$, satisfying

(a) $u_{\lambda} Y_{1}^{*}=0$ for all $\lambda \in \Lambda$.

(b) $u_{\lambda} u_{\lambda}^{*} \in \mathcal{D}$ and $u_{\lambda} u_{\mu}=^{*} 0$ for all $\lambda, \mu \in \Lambda$ with $\lambda \neq \mu$.

(c) $Y_{1}=\left[H_{0}^{2} Y_{1}\right]_{2}$.

(d) $\mathcal{K}_{1}=Y_{1} \oplus^{\text {row }}\left(\oplus_{\lambda \in \Lambda}^{\text {row }} H^{2} u_{\lambda}\right)$

Let

$$
Y=\left[Y_{1} \cap L^{p}(\mathcal{M}, \tau)\right]_{p}
$$

(i) From (a), definition of $Y$ and Lemma 2.5, we can conclude that

$$
u_{\lambda} Y^{*}=0 \text { for all } \lambda \in \Lambda \text {. }
$$

(ii) follows directly from (b), i.e.

$$
u_{\lambda} u_{\lambda}^{*} \in \mathcal{D} \text { and } u_{\lambda} u_{\mu}^{*}=0 \text { for all } \lambda, \mu \in \Lambda \text { with } \lambda \neq \mu \text {. }
$$

(iii) We want to show that $Y=\left[H_{0}^{2} Y\right]_{p}$. First we will show that

$$
\left[\left(H_{0}^{\infty} Y_{1}\right) \cap L^{p}(\mathcal{M}, \tau)\right]_{p} \subseteq\left[H_{0}^{\infty}\left(Y_{1} \cap L^{p}(\mathcal{M}, \tau)\right)\right]_{p}
$$


In fact, let $x \in Y_{1}$ and $h \in H_{0}^{\infty}$ such that $h x \in\left(H_{0}^{\infty} Y_{1}\right) \cap L^{p}(\mathcal{M}, \tau)$. We want to show that $h x \in\left[H_{0}^{\infty}\left(Y_{1} \cap L^{p}(\mathcal{M}, \tau)\right)\right]_{p}$. By Lemma 2.13, we can find a net $\left\{e_{\lambda}\right\}_{\lambda \in \Lambda}$ of projections in $\mathcal{D}$ such that $e_{\lambda} \rightarrow I$ in weak*-topology and $\tau\left(e_{\lambda}\right)<\infty$ for each $\lambda \in \Lambda$. By Lemma 2.6, we have

$$
\lim _{\lambda}\left\|e_{\lambda} h x-h x\right\|_{p}=0 .
$$

Thus, to show that $h x \in\left[H_{0}^{\infty}\left(Y_{1} \cap L^{p}(\mathcal{M}, \tau)\right)\right]_{p}$, it suffices to prove that $e_{\lambda} h x \in\left[H_{0}^{\infty}\left(Y_{1} \cap\right.\right.$ $\left.\left.L^{p}(\mathcal{M}, \tau)\right)\right]_{p}$ for each $\lambda \in \Lambda$. Fix a $\lambda_{0} \in \Lambda$. Then, for some positive number $q$ with $1 / p=$ $1 / 2+1 / q$, we have

$$
\lim _{\lambda}\left\|e_{\lambda_{0}} h e_{\lambda} x-e_{\lambda_{0}} h x\right\|_{p} \leq \lim _{\lambda}\left\|e_{\lambda_{0}} h\right\|_{q}\left\|e_{\lambda} x-x\right\|_{2}=0
$$

as $x \in Y_{1}$. Moreover, we have $e_{\lambda_{0}} h \in H_{0}^{\infty}$ and $e_{\lambda} x \in Y_{1} \cap L^{p}(\mathcal{M}, \tau)$, as $\left\|e_{\lambda} x\right\|_{p} \leq\left\|e_{\lambda}\right\|_{q}\|x\|_{2}<\infty$. Thus, $e_{\lambda_{0}} h e_{\lambda} x$ is in $H_{0}^{\infty}\left(Y_{1} \cap L^{p}(\mathcal{M}, \tau)\right)$ for each $\lambda \in \Lambda$. Whence, from (5.6), $e_{\lambda_{0}} h x$ is in $\left[H_{0}^{\infty}\left(Y_{1} \cap L^{p}(\mathcal{M}, \tau)\right)\right]_{p}$ for each $\lambda_{0} \in \Lambda$. Therefore, from $(\underline{5.6}), h x \in\left[H_{0}^{\infty}\left(Y_{1} \cap L^{p}(\mathcal{M}, \tau)\right)\right]_{p}$. Or

$$
\left[\left(H_{0}^{\infty} Y_{1}\right) \cap L^{p}(\mathcal{M}, \tau)\right]_{p} \subseteq\left[H_{0}^{\infty}\left(Y_{1} \cap L^{p}(\mathcal{M}, \tau)\right)\right]_{p}
$$

Now, we have

$$
\begin{aligned}
Y & =\left[Y_{1} \cap L^{p}(\mathcal{M}, \tau)\right]_{p} \\
& =\left[\left[H_{0}^{2} Y_{1}\right]_{2} \cap L^{p}(\mathcal{M}, \tau)\right]_{p} \\
& =\left[\left(H_{0}^{\infty} Y_{1}\right) \cap L^{p}(\mathcal{M}, \tau)\right]_{p} \\
& \subseteq\left[H_{0}^{\infty}\left(Y_{1} \cap L^{p}(\mathcal{M}, \tau)\right)\right]_{p} \\
& \subseteq\left[H_{0}^{\infty} Y\right]_{p} \subseteq Y .
\end{aligned}
$$

(by the definition of $Y$ )

Thus,

$$
Y=\left[H_{0}^{\infty} Y\right]_{p}
$$

(iv) We have only to show that

$$
\mathcal{K}=Y \oplus^{\text {row }}\left(\oplus_{\lambda \in \Lambda}^{\text {row }} H^{p} u_{\lambda}\right)
$$

By the definition of $Y$, we have

$$
Y=\left[Y_{1} \cap L^{p}(\mathcal{M}, \tau)\right]_{p}
$$

And from Lemma 5.3, we have

$$
H^{p} u_{\lambda}=\left[H^{2} u_{\lambda} \cap L^{p}(\mathcal{M}, \tau)\right]_{p}, \quad \forall \lambda \in \Lambda .
$$


Now, we have

$$
\begin{array}{rlr}
\mathcal{K} & =\left[\mathcal{K}_{1} \cap L^{p}(\mathcal{M}, \tau)\right]_{p} & \text { (by Proposition 5.1) } \\
& =\left[\left[\operatorname{span}\left\{Y_{1}, H^{2} u_{\lambda}: \lambda \in \Lambda\right\}\right]_{2} \cap L^{p}(\mathcal{M}, \tau)\right]_{p} & \text { (by the definition of row sum of subspaces) } \\
& =\left[\operatorname{span}\left\{Y_{1}, H^{2} u_{\lambda}: \lambda \in \Lambda\right\} \cap L^{p}(\mathcal{M}, \tau)\right]_{p} & \text { (by Proposition [5.2) } \\
& =\left[\operatorname{span}\left\{Y_{1} \cap L^{p}(\mathcal{M}, \tau), H^{2} u_{\lambda} \cap L^{p}(\mathcal{M}, \tau): \lambda \in \Lambda\right\}\right]_{p} & \text { (by (a) and (b)) } \\
& =\left[\operatorname{span}\left\{Y, H^{p} u_{\lambda}: \lambda \in \Lambda\right\}\right]_{p} & \text { (by (5.9) and (55.10) }) \\
& =Y \oplus^{\text {row }}\left(\oplus_{\lambda \in \Lambda}^{\text {row }} H^{p} u_{\lambda}\right),
\end{array}
$$

where the last equation follows from the definition of the row sum of subspaces.

As a summary, from (5.3), (5.4), (5.8), and (5.11), $Y$ and $\left\{u_{\lambda}\right\}_{\lambda \in \Lambda}$ have desired properties. This ends the proof of the theorem.

Corollary 5.5. Let $\mathcal{M}$ be a von Neumann algebra with a faithful, normal, semifinite tracial weight $\tau$.

(i) Let $0<p<\infty$. If $\mathcal{K}$ is a closed subspace of $L^{p}(\mathcal{M}, \tau)$ such that $\mathcal{M K} \subseteq \mathcal{K}$, then there exists a projection $q \in \mathcal{M}$ such that $\mathcal{K}=L^{p}(\mathcal{M}, \tau) q$.

(ii) If $\mathcal{K}$ is a weak $k^{*}$-closed subspace of $\mathcal{M}$ such that $\mathcal{M K} \subseteq \mathcal{K}$, then there exists a projection $q \in \mathcal{M}$ such that $\mathcal{K}=\mathcal{M} q$.

Proof. (i) Note that $\mathcal{M}$ itself is a semifinite subdiagonal subalgebra of $\mathcal{M}$. Let $H^{\infty}=\mathcal{M}$. Then $\mathcal{D}=\mathcal{M}$ and $\Phi$ is the identity map from $\mathcal{M}$ to $\mathcal{M}$. Hence $H_{0}^{\infty}=\{0\}$ and $H^{p}=L^{p}(\mathcal{M}, \tau)$.

Assume that $\mathcal{K}$ is a closed subspace of $L^{p}(\mathcal{M}, \tau)$ such that $\mathcal{K} \mathcal{M} \subseteq \mathcal{K}$. From Theorem 4.6 and Theorem 5.4,

$$
\mathcal{K}=Y \oplus^{\text {row }}\left(\oplus_{\lambda \in \Lambda}^{\text {row }} H^{p} u_{\lambda}\right),
$$

where $Y$ and the $\left\{u_{\lambda}\right\}_{\lambda \in \Lambda}$ satisfy the conditions in Theorem 4.6 and Theorem 5.4.

From the fact that $H_{0}^{\infty}=\{0\}$, we know that $Y=\{0\}$. Since $\mathcal{D}=\mathcal{M}$, we know that

$$
H^{p} u_{\lambda}=L^{p}(\mathcal{M}, \tau) u_{\lambda}=L^{p}(\mathcal{M}, \tau) u_{\lambda} u_{\lambda}^{*} u_{\lambda} \subseteq L^{p}(\mathcal{M}, \tau) u_{\lambda}^{*} u_{\lambda} \subseteq L^{p}(\mathcal{M}, \tau) u_{\lambda}=H^{p} u_{\lambda} .
$$

So $H^{p} u_{\lambda}=L^{p}(\mathcal{M}, \tau) u_{\lambda}^{*} u_{\lambda}$ and

$$
\mathcal{K}=Y \oplus^{\text {row }}\left(\oplus_{\lambda \in \Lambda}^{\text {row }} H^{p} u_{\lambda}\right)=\left(\oplus_{\lambda \in \Lambda}^{\text {row }} L^{p}(\mathcal{M}, \tau) u_{\lambda}^{*} u_{\lambda}\right)=L^{p}(\mathcal{M}, \tau)\left(\sum_{\lambda \in \Lambda} u_{\lambda}^{*} u_{\lambda}\right)=L^{p}(\mathcal{M}, \tau) q
$$

where $q=\sum_{\lambda \in \Lambda} u_{\lambda}^{*} u_{\lambda}$ is a projection in $\mathcal{M}$. This ends the proof of (i).

(ii) The proof is similar to (i).

\section{Invariant Subspaces for Analytic Crossed Products}

6.1. Crossed product of a von Neumann algebra $\mathcal{M}$ by an action $\alpha$. Let $\mathcal{M}$ be a von Neumann algebra with a semifinite, faithful, normal tracial state $\tau$. Let $\alpha$ be a trace-preserving *-automorphism of $\mathcal{M}$ (so $\left.\tau(\alpha(x))=\tau(x), \forall x \in \mathcal{M}^{+}\right)$.

We let $l^{2}(\mathbb{Z})$ be the Hilbert space consisting of complex-valued functions $f$ on $\mathbb{Z}$ such that $\sum_{m \in \mathbb{Z}}|f(m)|^{2}<\infty$. We denote by $\left\{e_{n}\right\}_{n \in \mathbb{Z}}$ the orthonormal basis of $l^{2}(\mathbb{Z})$ determined by 
$e_{n}(m)=\delta(n, m)$. We also denote by $\lambda: \mathbb{Z} \rightarrow B\left(l^{2}(\mathbb{Z})\right)$ the left regular representation of $\mathbb{Z}$ on $l^{2}(\mathbb{Z})$, i.e. each $\lambda(n)$ is determined by $\lambda(n)\left(e_{m}\right)=e_{m+n}$.

Let $\mathcal{H}=L^{2}(\mathcal{M}, \tau) \otimes l^{2}(\mathbb{Z})$. Then $\mathcal{H}$ can also be written as $\oplus_{m \in \mathbb{Z}} L^{2}(\mathcal{M}, \tau) \otimes e_{m}$. Consider representations $\Psi$ and $\Lambda$ of $\mathcal{M}$ and $\mathbb{Z}$, respectively, on $\mathcal{H}$, defined by

$$
\begin{array}{lc}
\Psi(x)\left(\xi \otimes e_{m}\right)=\left(\alpha^{-m}(x) \xi\right) \otimes e_{m}, & \forall x \in \mathcal{M}, \forall \xi \in L^{2}(\mathcal{M}, \tau), \forall m \in \mathbb{Z} \\
\Lambda(n)\left(\xi \otimes e_{m}\right)=\xi \otimes\left(\lambda(n) e_{m}\right), & \forall n, m \in \mathbb{Z}
\end{array}
$$

It can be verified that

$$
\Lambda(n) \Psi(x) \Lambda(-n)=\Psi\left(\alpha^{n}(x)\right), \quad \forall x \in \mathcal{M}, \forall n \in \mathbb{Z} .
$$

Then the crossed product of $\mathcal{M}$ by an action $\alpha$, denoted by $\mathcal{M} \rtimes_{\alpha} \mathbb{Z}$, is the von Neumann algebra generated by $\Psi(\mathcal{M})$ and $\Lambda(\mathbb{Z})$ in $B(\mathcal{H})$. If no confusion arises, we will identify $\mathcal{M}$ with its image $\Psi(\mathcal{M})$ in $\mathcal{M} \rtimes_{\alpha} \mathbb{Z}$.

It is well known (for example, see Chapter 13 in [19]) that there exists a faithful, normal conditional expectation $\Phi$ from $\mathcal{M} \rtimes_{\alpha} \mathbb{Z}$ onto $\mathcal{M}$ such that

$$
\Phi\left(\sum_{n=-N}^{N} \Lambda(n) \Psi\left(x_{n}\right)\right)=x_{0}, \quad \text { where } x_{n} \in \mathcal{M} \text { for all }-N \leq n \leq N .
$$

Moreover, there exists a semifinite, faithful, normal, extended tracial weight, still denoted by $\tau$, on $\mathcal{M} \rtimes_{\alpha} \mathbb{Z}$ satisfying

$$
\tau(y)=\tau(\Phi(y)), \quad \text { for every positive element } y \text { in } \mathcal{M} \rtimes_{\alpha} \mathbb{Z} .
$$

EXAMPLE 6.1. Let $\mathcal{M}=l^{\infty}(\mathbb{Z})$ be an abelian von Neumann algebra with a semifinite, faithful, normal tracial weight $\tau$, determined by

$$
\tau(f)=\sum_{m \in \mathbb{Z}} f(m), \quad \text { for every positive element } f \in l^{\infty}(\mathbb{Z}) .
$$

Let $\alpha$ be an action on $l^{\infty}(\mathbb{Z})$, defined by

$$
\alpha(f)(m)=f(m-1), \quad \text { for every element } f \in l^{\infty}(\mathbb{Z}) .
$$

It is not hard to verify (for example see Proposition 8.6.4 in $[\mathbf{1 9}]$ ) that $l^{\infty}(\mathbb{Z}) \rtimes_{\alpha} \mathbb{Z}$ is a type $I_{\infty}$ factor. Thus $l^{\infty}(\mathbb{Z}) \rtimes_{\alpha} \mathbb{Z} \simeq B(\mathcal{H})$ for some separable Hilbert space $\mathcal{H}$.

6.2. Invariant subspace for crossed products. From the construction of crossed product, we have the following result immediately (also see Section 3 in [1]).

LEMMA 6.2. Let $\mathcal{M} \rtimes_{\alpha} \mathbb{Z}_{+}$be a weak *-closed non-self-adjoint subalgebra generated by

$$
\{\Lambda(n) \Psi(x): x \in \mathcal{M}, n \geq 0\}
$$

in $\mathcal{M} \rtimes_{\alpha} \mathbb{Z}$. Then the following statements are true:

(i) $\mathcal{M} \rtimes_{\alpha} \mathbb{Z}_{+}$is a semifinite subdiagonal subalgebra with respect to $\left(\mathcal{M} \rtimes_{\alpha} \mathbb{Z}, \Phi\right)$. (Such $\mathcal{M} \rtimes_{\alpha} \mathbb{Z}_{+}$is called an analytic crossed product and will be denoted by $H^{\infty}$.) 
(ii) $H_{0}^{\infty}=\operatorname{ker}(\Phi) \cap H^{\infty}$ is a weak *-closed nonself-adjoint subalgebra generated by

$$
\{\Lambda(n) \Psi(x): x \in \mathcal{M}, n>0\}
$$

in $\mathcal{M} \rtimes_{\alpha} \mathbb{Z}$ satisfying

$$
H_{0}^{\infty}=\Lambda(1) H^{\infty}
$$

(iii) $H^{\infty} \cap\left(H^{\infty}\right)^{*}=\mathcal{M}$.

Following the notation in Section 6.1, our next result characterizes invariant subspaces in a crossed product of a semifinite von Neumann algebra $\mathcal{M}$ by a tracing-preserving action $\alpha$.

THEOREM 6.3. Let $\mathcal{M}$ be a von Neumann algebra with a semifinite, faithful, normal tracial weight $\tau$, and $\alpha$ be a trace-preserving *-automorphism of $\mathcal{M}$. Denote by $\mathcal{M} \rtimes_{\alpha} \mathbb{Z}$ the crossed product of $\mathcal{M}$ by an action $\alpha$, and still denote by $\tau$ a semifinite, faithful, normal, extended tracial weight on $\mathcal{M} \rtimes_{\alpha} \mathbb{Z}$.

Let $H^{\infty}$ be a weak $*$-closed non-self-adjoint subalgebra generated by $\{\Lambda(n) \Psi(x): x \in \mathcal{M}, n \geq$ $0\}$ in $\mathcal{M} \rtimes_{\alpha} \mathbb{Z}$, be a semifinite subdiagonal subalgebra of $\mathcal{M} \rtimes_{\alpha} \mathbb{Z}$. Then the following statements are true.

(i) Let $0<p<\infty$. Assume that $\mathcal{K}$ is a closed subspace of $L^{p}\left(\mathcal{M} \rtimes_{\alpha} \mathbb{Z}, \tau\right)$ such that $H^{\infty} \mathcal{K} \subseteq \mathcal{K}$. Then there exist a projection $q$ in $\mathcal{M}$ and a family $\left\{u_{\lambda}\right\}_{\lambda \in \Lambda}$ of partial isometries in $\mathcal{M} \rtimes_{\alpha} \mathbb{Z}$ satisfying

(a) $u_{\lambda} q=0$ for all $\lambda \in \Lambda$;

(b) $u_{\lambda} u_{\lambda}^{*} \in \mathcal{M}$ and $u_{\lambda} u_{\mu}^{*}=0$ for all $\lambda, \mu \in \Lambda$ with $\lambda \neq \mu$;

(c) $\mathcal{K}=\left(L^{p}\left(\mathcal{M} \rtimes_{\alpha} \mathbb{Z}, \tau\right) q\right) \oplus^{\text {row }}\left(\oplus_{\lambda \in \Lambda}^{\text {row }} H^{p} u_{\lambda}\right)$.

(ii) Assume that $\mathcal{K}$ is a weak $*$-closed subspace of $\mathcal{M} \rtimes_{\alpha} \mathbb{Z}$ such that $H^{\infty} \mathcal{K} \subseteq \mathcal{K}$. Then there exist a projection $q$ in $\mathcal{M}$ and a family $\left\{u_{\lambda}\right\}_{\lambda \in \Lambda}$ of partial isometries in $\mathcal{M} \rtimes_{\alpha} \mathbb{Z}$ satisfying

(a) $u_{\lambda} q=0$ for all $\lambda \in \Lambda$;

(b) $u_{\lambda} u_{\lambda}^{*} \in \mathcal{M}$ and $u_{\lambda} u_{\mu}^{*}=0$ for all $\lambda, \mu \in \Lambda$ with $\lambda \neq \mu$;

(c) $\mathcal{K}=\left(\left(\mathcal{M} \rtimes_{\alpha} \mathbb{Z}\right) q\right) \oplus^{\text {row }}\left(\oplus_{\lambda \in \Lambda}^{\text {row }} H^{\infty} u_{\lambda}\right)$.

Proof. (i) From Theorem 4.6 and Theorem 5.4 ,

$$
\mathcal{K}=Y \oplus^{\text {row }}\left(\oplus_{\lambda \in \Lambda}^{\text {row }} H^{p} u_{\lambda}\right),
$$

where $Y$ is a closed subspace of $\mathcal{M} \rtimes_{\alpha} \mathbb{Z}$ and $\left\{u_{\lambda}\right\}_{\lambda \in \Lambda}$ is a family of partial isometries in $\mathcal{M} \rtimes_{\alpha} \mathbb{Z}$ satisfying

(a) $u_{\lambda} Y^{*}=0$ for all $\lambda \in \Lambda$;

$\left(\mathrm{b}_{1}\right) u_{\lambda} u_{\lambda}^{*} \in \mathcal{M}$ and $u_{\lambda} u_{\mu}^{*}=0$ for all $\lambda, \mu \in \Lambda$ with $\lambda \neq \mu$;

$\left(c_{1}\right) Y=\left[H_{0}^{\infty} Y\right]_{p}$.

From $\left(\mathrm{c}_{1}\right)$ and Lemma 6.2, we have

$$
Y=\left[H_{0}^{\infty} Y\right]_{p}=\left[\Lambda(1) H^{\infty} Y\right]_{p} \subseteq \Lambda(1) Y .
$$

So, $Y$ is a left $\mathcal{M} \rtimes_{\alpha} \mathbb{Z}$-invariant subspace of $L^{p}\left(\mathcal{M} \rtimes_{\alpha} \mathbb{Z}, \tau\right)$. From Corollary 5.5, there exists a projection $q$ in $\mathcal{M}$ such that $Y=L^{p}\left(\mathcal{M} \rtimes_{\alpha} \mathbb{Z}, \tau\right) q$. Therefore, we have 
(a) $u_{\lambda} q=0$ for all $\lambda \in \Lambda$;

(b) $u_{\lambda} u_{\lambda}^{*} \in \mathcal{M}$ and $u_{\lambda} u_{\mu}^{*}=0$ for all $\lambda, \mu \in \Lambda$ with $\lambda \neq \mu$;

(c) $\mathcal{K}=\left(L^{p}\left(\mathcal{M} \rtimes_{\alpha} \mathbb{Z}, \tau\right) q\right) \oplus^{\text {row }}\left(\oplus_{\lambda \in \Lambda}^{\text {row }} H^{p} u_{\lambda}\right)$.

This ends the proof of (i).

(ii) The proof is similar to (i).

6.3. Invariant subspaces for Schatten $p$-classes. Let $\mathcal{H}$ be an infinite dimensional separable Hilbert space with an orthonormal base $\left\{e_{m}\right\}_{m \in \mathbb{Z}}$. Let $\tau=\operatorname{Tr}$ be the usual trace on $B(\mathcal{H})$, i.e.

$$
\tau(x)=\sum_{i \in \mathbb{Z}}\left\langle x e_{m}, e_{m}\right\rangle, \quad \text { for all positive } x \text { in } B(\mathcal{H}) .
$$

Then $B(\mathcal{H})$ is a von Neumann algebra with a semifinite, faithful, normal tracial weight $\tau$. For each $0<p<\infty$, the Schatten $p$-class $S^{p}(\mathcal{H})$ is the associated non-commuative $L^{p}$-space $L^{p}(B(\mathcal{H}), \tau)$.

Let

$$
\mathcal{A}=\left\{x \in B(\mathcal{H}):\left\langle x e_{m}, e_{n}\right\rangle=0, \quad \forall n<m\right\}
$$

be the lower triangular subalgebra of $B(\mathcal{H})$. From Example 6.1, $B(\mathcal{H})$ can also be realized as a crossed product $l^{\infty}(\mathbb{Z}) \rtimes_{\alpha} \mathbb{Z}$ of $l^{\infty}(\mathbb{Z})$ by an action $\alpha$, where the action $\alpha$ is determined by

$$
\alpha(f)(m)=f(m-1), \quad \forall f \in l^{\infty}(\mathbb{Z}) .
$$

Moreover, it can be verified quickly that $\mathcal{A}$, as a subalgebra of $B(\mathcal{H})$, is $l^{\infty}(\mathbb{Z}) \rtimes_{\alpha} \mathbb{Z}_{+}$(see Lemma 6.2) is a semifinite subdiagonal subalgebra of $l^{\infty}(\mathbb{Z}) \rtimes_{\alpha} \mathbb{Z}$ (see Example 2.6 in [25]). Thus from Theorem 6.3, we have the following statements.

Corollary 6.4. Let $\mathcal{H}$ be a separable Hilbert space with an orthonormal base $\left\{e_{m}\right\}_{m \in \mathbb{Z}}$. Let $H^{\infty}$ be the lower triangular subalgebra of $B(\mathcal{H})$, i.e.

$$
H^{\infty}=\left\{x \in B(\mathcal{H}):\left\langle x e_{m}, e_{n}\right\rangle=0, \quad \forall n<m\right\} .
$$

Let $\mathcal{D}=H^{\infty} \cap\left(H^{\infty}\right)^{*}$ be the diagonal subalgebra of $B(\mathcal{H})$.

(i) For each $0<p<\infty$, let $S^{p}(\mathcal{H})$ be the Schatten $p$-class. Assume that $\mathcal{K}$ is a closed subspace of $S^{p}(\mathcal{H})$ such that $H^{\infty} \mathcal{K} \subseteq \mathcal{K}$. Then there exist a projection $q$ in $\mathcal{D}$ and a family $\left\{u_{\lambda}\right\}_{\lambda \in \Lambda}$ of partial isometries in $B(\mathcal{H})$ satisfying

(a) $u_{\lambda} q=0$ for all $\lambda \in \Lambda$;

(b) $u_{\lambda} u_{\lambda}^{*} \in \mathcal{D}$ and $u_{\lambda} u_{\mu}^{*}=0$ for all $\lambda, \mu \in \Lambda$ with $\lambda \neq \mu$;

(c) $\mathcal{K}=\left(S^{p}(\mathcal{H}) q\right) \oplus^{\text {row }}\left(\oplus_{\lambda \in \Lambda}^{\text {row }} H^{p} u_{\lambda}\right)$.

(ii) Assume that $\mathcal{K}$ is a weak $*$-closed subspace of $B(\mathcal{H})$ such that $H^{\infty} \mathcal{K} \subseteq \mathcal{K}$. Then there exist a projection $q$ in $\mathcal{D}$ and a family $\left\{u_{\lambda}\right\}_{\lambda \in \Lambda}$ of partial isometries in $B(\mathcal{H})$ satisfying

(a) $u_{\lambda} q=0$ for all $\lambda \in \Lambda$;

(b) $u_{\lambda} u_{\lambda}^{*} \in \mathcal{D}$ and $u_{\lambda} u_{\mu}^{*}=0$ for all $\lambda, \mu \in \Lambda$ with $\lambda \neq \mu$;

(c) $\mathcal{K}=(B(\mathcal{H}) q) \oplus^{\text {row }}\left(\oplus_{\lambda \in \Lambda}^{\text {row }} H^{\infty} u_{\lambda}\right)$.

REMARK 6.5. Let $0<p<\infty$. If $q$ is a projection in $\mathcal{D}$ such that $S^{p}(\mathcal{H}) q \subseteq H^{p}$, then $q=0$. 
The the next result follows directly from Corollary 6.4.

Corollary 6.6. Let $\mathcal{H}$ be a separable Hilbert space with an orthonormal base $\left\{e_{m}\right\}_{m \in \mathbb{Z}}$. Let $H^{\infty}$ be the lower triangular subalgebra of $B(\mathcal{H})$, i.e.

$$
H^{\infty}=\left\{x \in B(\mathcal{H}):\left\langle x e_{m}, e_{n}\right\rangle=0, \quad \forall n<m\right\} .
$$

Let $\mathcal{D}=H^{\infty} \cap\left(H^{\infty}\right)^{*}$ be the diagonal subalgebra of $B(\mathcal{H})$.

(i) For each $0<p<\infty$, if $\mathcal{K}$ is a closed subspace of $H^{p}$ such that $H^{\infty} \mathcal{K} \subseteq \mathcal{K}$, then there exists a family $\left\{u_{\lambda}\right\}_{\lambda \in \Lambda}$ of partial isometries in $H^{\infty}$ satisfying

(a) $u_{\lambda} u_{\lambda}^{*} \in \mathcal{D}$ and $u_{\lambda} u_{\mu}^{*}=0$ for all $\lambda, \mu \in \Lambda$ with $\lambda \neq \mu$;

(b) $\mathcal{K}=\oplus_{\lambda \in \Lambda}^{\text {row }} H^{p} u_{\lambda}$.

(ii) Assume that $\mathcal{K}$ is a weak *-closed subspace of $H^{\infty}$ such that $H^{\infty} \mathcal{K} \subseteq \mathcal{K}$. Then there exists a family $\left\{u_{\lambda}\right\}_{\lambda \in \Lambda}$ of partial isometries in $H^{\infty}$ satisfying

(a) $u_{\lambda} u_{\lambda}^{*} \in \mathcal{D}$ and $u_{\lambda} u_{\mu}^{*}=0$ for all $\lambda, \mu \in \Lambda$ with $\lambda \neq \mu$;

(b) $\mathcal{K}=\oplus_{\lambda \in \Lambda}^{\text {row }} H^{\infty} u_{\lambda}$.

REMARK 6.7. Similar results hold when $H^{\infty}$ is the upper triangular subalgebra of $B(\mathcal{H})$.

\section{References}

[1] W. B. Arveson, Analyticity in operator algebras, Amer. J. Math. 89 (1967) 578-642.

[2] T. Bekjan, Noncommutative Hardy space associated with semi-finite subdiagonal algebras, J. Math. Anal. Appl. 429 (2015), no. 2, 13471369.

[3] T. N. Bekjan and Q. Xu, Riesz and Szegö type factorizations for noncommutative Hardy spaces, J. Operator Theory, 62 (2009) 215-231.

[4] T. N. Bekjan, Noncommutative symmetric Hardy spaces, Integr. Equ. Oper. Theory 81 (2015) 191-212.

[5] T. N. Bekjan, Noncommutative Hardy space associated with semi-finite subdiagonal algebras, J. Math. Anal. Appl. 429 (2015), no. 2, 1347-1369.

[6] A. Beurling, On two problems concerning linear transformations in Hilbert space, Acta Math. 81 (1949) 239-255.

[7] D. Blecher and L. E. Labuschagne, A Beurling theorem for noncommutative $L^{p}$, J. Operator Theory, 59 (2008) 29-51.

[8] S. Bochner, Generalized conjugate and analytic functions without expansions, Proc. Nat. Acad. Sci. U.S.A. 45 (1959) 855-857.

[9] P. Dodds and T. Dodds, Some properties of symmetric operator spaces, Proc. Centre Math. Appl. Austral. Nat. Univ., 29, Austral. Nat. Univ., Canberra, 1992.

[10] P. Dodds, T. Dodds and B. Pagter, Noncommutative Banach function spaces, Mathematische Zeitschrift, 201 (1989) 583-597.

[11] P. Dodds, T. Dodds and B. Pagter, Noncommutative Köthe duality, Trans. Amer. Math. Soc. 339 (1993) 717-750.

[12] R. Exel, Maximal subdiagonal algebras, Amer. J. Math. 110 (1988) 775-782.

[13] T. Fack and H. Kosaki, Generalized s-numbers of $\tau$-measurable operators, Pacific J. Math. 2 (1986) $269-300$.

[14] J. Fang, D. Hadwin, E. Nordgren and J. Shen, Tracial gauge norms on finite von Neumann algebras satisfying the weak Dixmier property, J. Funct. Anal. 255 (2008) 142-183.

[15] P. Halmos, Shifts on Hilbert spaces, J. Reine Angew. Math. 208 (1961) 102-112.

[16] H. Helson, Lectures on Invariant Subspaces, Academic Press, New York-London, 1964.

[17] H. Helson and D. Lowdenslager, Prediction theory and Fourier series in several variables, Acta Math. 99 (1958) 165-202. 
[18] K. Hoffman, Analytic functions and logmodular Banach algebras, Acta Math. 108 (1962) 271-317.

[19] R. Kadison and J. Ringrose, Fundamentals of the thoery of operator algebras, volume II, advanced theory, Academic Press, Inc, (1986).

[20] R. A. Kunze, $L^{p}$-Fourier transforms on locally compact unimodular groups, Trans. Amer. Math. Soc. 89 (1958) 519-540.

[21] G. Ji, Maximality of semi-finite subdiagonal algebras, J. Shaanxi Normal Univ. Sci. Ed. 28 (2000) 15-17.

[22] M. Junge and D. Sherman, Noncommutative $L^{p}$-modules, J. Operator Theory 53 (2005) 3-34.

[23] M. Marsalli and G. West, Noncommutative $H^{p}$ spaces, J. Operator Theory 40 (1998) 339-355.

[24] C. A. McCarthy, $C_{p}$, Israel J. Math. 5 (1967) 249-271.

[25] M. McAsey, P. Muhly and K. Saito, Nonselfadjoint Crossed Products (Invariant Subspaces and Maximality), Transactions of the American Mathematical Society, Vol. 248, No. 2 (Mar., 1979), pp. 381 -409.

[26] T. Nakazi and Y. Watatani, Invariant subspace theorems for subdiagonal algebras, J. Operator Theory 37(1997), 379-395.

[27] E. Nelson, Notes on noncommutative integration, J. Funct. Anal. 15 (1974) 103-116.

[28] J. von Neumann, Some matrix-inequalities and metrization of matric-space, Tomsk Univ. Rev. 1 (1937) 286-300.

[29] G. Pisier and Q. Xu, Noncommutative $L^{p}$-spaces, Handbook of the geometry of Banach spaces, NorthHolland, Amsterdam, 2 (2003) 1459-1517.

[30] Sakai, $C^{*}$-algebras and $W^{*}$-algebras, Springer, 1971.

[31] K. S. Saito, A note on invariant subspaces for finite maximal subdiagonal algebras, Proc. Amer. Math. Soc. 77 (1979) 348-352.

[32] K. S. Saito, A simple approach to the invariant subspace structure of analytic crossed products, J. Operator Theory 27 (1992), no. 1, 169-177.

[33] I. Segal, A noncommutative extension of abstract integration, Ann. Math. 57 (1952) 401-457.

[34] B. Simon, Trace ideals and their applications, London Mathematical Society Lecture Note Series, vol. 35, Cambridge University Press, Cambridge-New York, 1979.

[35] T. P. Srinivasan, Simply invariant subspaces, Bull. Amer. Math. Soc. 69 (1963) 706-709.

[36] T. Srinivasan and J. K. Wang, Weak*-Dirichlet algebras, Proceedings of the International Symposium on Function Algebras, Tulane University, 1965 (Chicago), Scott-Foresman, 1966, 216-249.

[37] M. Takesaki, Theory of Operator Algebras I, Springer, 1979.

[38] Q. Xu, On the maximality of subdiagonal algebras, J. Operator Theory 54 (2005), no. 1, 137-146.

[39] Q. Xu Operator spaces and noncommutative $L^{p}$, The part on noncommutative $L^{p}$-spaces Lectures in the Summer School on Banach spaces and Operator spaces, Nankai University China July 16 - July 20, 2007.

[40] F. Yeadon, Noncommutative $L^{p}$-spaces, Math. Proc. Cambridge Philos. Soc. 77 (1975) 91-102.

Lauren Sager, Demartment of Mathematics and Statistics, University of New Hampshire, DURHAM, NH 03824; EMAIL: LBQ32@WILDCATS.UNH.EDU 